\title{
L'autobiografia del chirurgo Tommaso Rima (1775-1843)
}

\author{
«Necrologia del D. ${ }^{r}$ T. Rima» \\ edita da Luigi Belloni, Milano
}

La storia della chirurgia riserva un posto d'onore a Tommaso Rima (1775-1843) pei suoi studi sulla patologia e la terapia delle vene varicose e soprattutto per la descrizione del reflusso sanguigno nelle varici dell'arto inferiore.

Benchè tali studi abbiano avuto una certa risonanza nell'Ottocento furono tra l'altro recensiti sulla Gazette Médicale de Paris ${ }^{1}$ ed esposti nei trattati di patologia chirurgica di Mighelangelo Asson (1802-1877)² e di Auguste Nélaton (1807-1873) - i concetti sulla genesi e la terapia delle varici propugnati dal Rima ebbero universale accoglienza in chirurgia soltanto alla fine del secolo, dopo la comparsa (1891) del lavoro 4 di Friedrich Trendelenburg (1844-1924). Oggi il reflusso sanguigno nelle varici dell'arto inferiore viene per lo più indicato quale «fenomeno di Trendelenburg», mentre il nome del Rima è generalmente dimenticato.

La rivendicazione a questo chirurgo, nativo di Mosogno nel cantone Ticino, fu compiuta da Davide Giondano (n. 1864), come il Rima chirurgo primario all'Ospedale civile di Venezia, che da «buon moderno» ha criticamente valutato l'opera chirurgica del suo predecessore e ne ha steso la biografia ${ }^{5}$. In questo lavoro il Giondano fu soccorso dal Rima stesso, che aveva scritto un'autobiografia in terza persona, la Necrologia del $D .^{r} T$. Rima.

Il manoscritto autografo, donato dal Rima all'allievo M. Asson', servì a quest'ultimo per stendere 1'Elogio del maestro, pronunciato all'Ateneo Veneto il 6 marzo 18437, e a Luigi Nardo (1806-1870), segretario della Direzione medica dell'ospedale di Venezia, per preparare il discorso funebre letto nella chiesa interna dell'ospedale stesso il 27 marzo $1843^{8}$. Per interessamento del NARDo, il manoscritto fu depositato presso l'archivio della direzione dell'ospedale di Venezia, ed ivi rimase finché M. Asson ritenne più opportuno affidarlo a un discendente del Rima che aveva abbracciato la carriera medica: Orazio Pinelli $^{9}$. Di qui passò a D. Giondano, che il 20 febbraio 1947 - svolgendosi alla Biblioteca Cantonale di Lugano l'esposizione bibliografica «Ricerche, scoperte e invenzioni di medici svizzeri ${ }^{10}$ - lo volle donare con una nobile lettera alla Biblioteca del Cantone che al RimA aveva dato i natali. Sulla falsariga della prima parte del manoscritto sono stese anche le notizie biografiche sul Rima pubblicate da Giovanni Alfonso Oldelli (1733-1821) ${ }^{13}$.

Il manoscritto è costituito da 32 fogli di robusta carta a mano grigio-azzurrognola delle dimensioni di cm $21 \times 30$ circa, scritti tanto al recto che al verso. L'autore piegò i fogli secondo la dimensione maggiore, in modo da utilizzare soltanto una metà per il testo e da 
lasciare l'altra metà per le eventuali note, aggiunte e correzioni. I 32 fogli della «Necrologia» sono ricoperti da un foglio bianco ingiallito dal tempo, meno robusto, contenente alcune scritte d'altra mano che si riferiscono ai cambiamenti di proprietà del manoscritto: «al N. ${ }^{\circ}$ 2292, 25 novembre 1843, II 3 / Manoscritto autografo / delle Memorie concernenti la vita / del D. ${ }^{r}$ Tommaso Rima / regalato dal D. ${ }^{r}$ Michelangelo Asson / che dal Rima medesimo l'ebbe in dono»; seguono di mano di D. Grondano: «ad Orazio Pinelli/che lo donò a Davide Giordano» e il timbro a inchiostro «D. ${ }^{r}$ D. Giordano / S. Leonardo $1574-V e n e z i a »$.

Il manoscritto è autografo, e la grafia non è sempre facile, soprattutto negli ultimi fogli : molti fra quanti utilizzarono il manoscritto incorsero facilmente in errori di trascrizione, soprattutto a carico dei nomi propri. Per evitare questo inconveniente, nei limiti del possibile, ho controllato i singoli nomi: quando la ricerca riuscì infruttuosa, fu trascritta la lezione più probabile aggiungendo un punto d'interrogazione. La trascrizione fu eseguita cercando di rispettare la punteggiatura e, fin dove fu possibile, l'uso delle maiuscole.

Le note dell'Autore furono incorporate nel testo tra parentesi quadre, mentre quelle dell'editore trovarono posto alla fine del lavoro. In esse ho dato la prevalenza a quelle di carattere bio-bibliografico, con maggior riferimento alla storia della medicina che a quella dell'epopea napoleonica. Rimando infatti alle due classiche opere del ZANoLI e del TurotTI sulla storia delle truppe italiane agli ordini del grande Còrso ${ }^{11}$, il lettore che sia particolarmente interessato al lato militare dell'autobiografia del Rima.

L. B.

\section{Necrologia del D. ${ }^{r}$ T. Rima}

Se l'istoria parlare non dovesse se non che di quegli individui che per meriti straordinarj si resero degni di avervi un posto distinto, il numero degli uomini veramente illustri sarebbe assai ristretto, nè il D. ${ }^{\mathrm{r}}$ RIMA si lascerebbe ingannare dall'amor proprio per credersi meritevole d'esser tra questi annoverato. Ma non così va la cosa. Il compilatore delle necrologie ha interesse che il volume si accresca, e facilmente accoglie che gli offre materiali: parenti o amici d'un defonto appena al di sopra della mediocrità, credono parteciparne alla gloria ricordandolo ai posteri, e ne magnificano senza limite i pregi, descrivendo giganti quelli che appena s'innalzano sopra i pigmei.

Il sottoscritto ha corso una carriera che per vicende ora tristissime, ora fauste potrà sotto vario aspetto esser messo a notizia de' suoi contemporanei. Al meno svisarne la verità storica egli ne lascia uniti i dati genuini all'oggetto che chiunque volesse prendersi l'incarico di scrivere di lui, ne abbia gli elementi vergati con la sincerità d'uno che si sente vicino alla tomba, e che non ebbe mai l'abitudine di mentire.

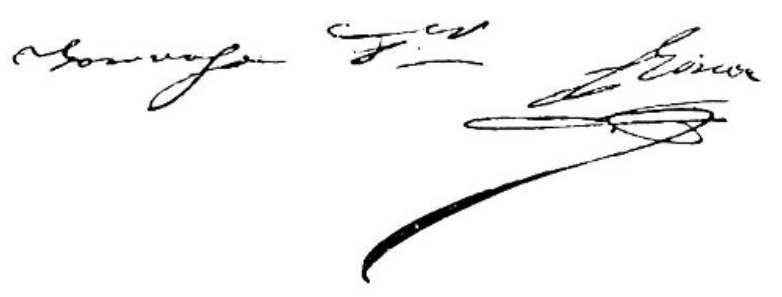




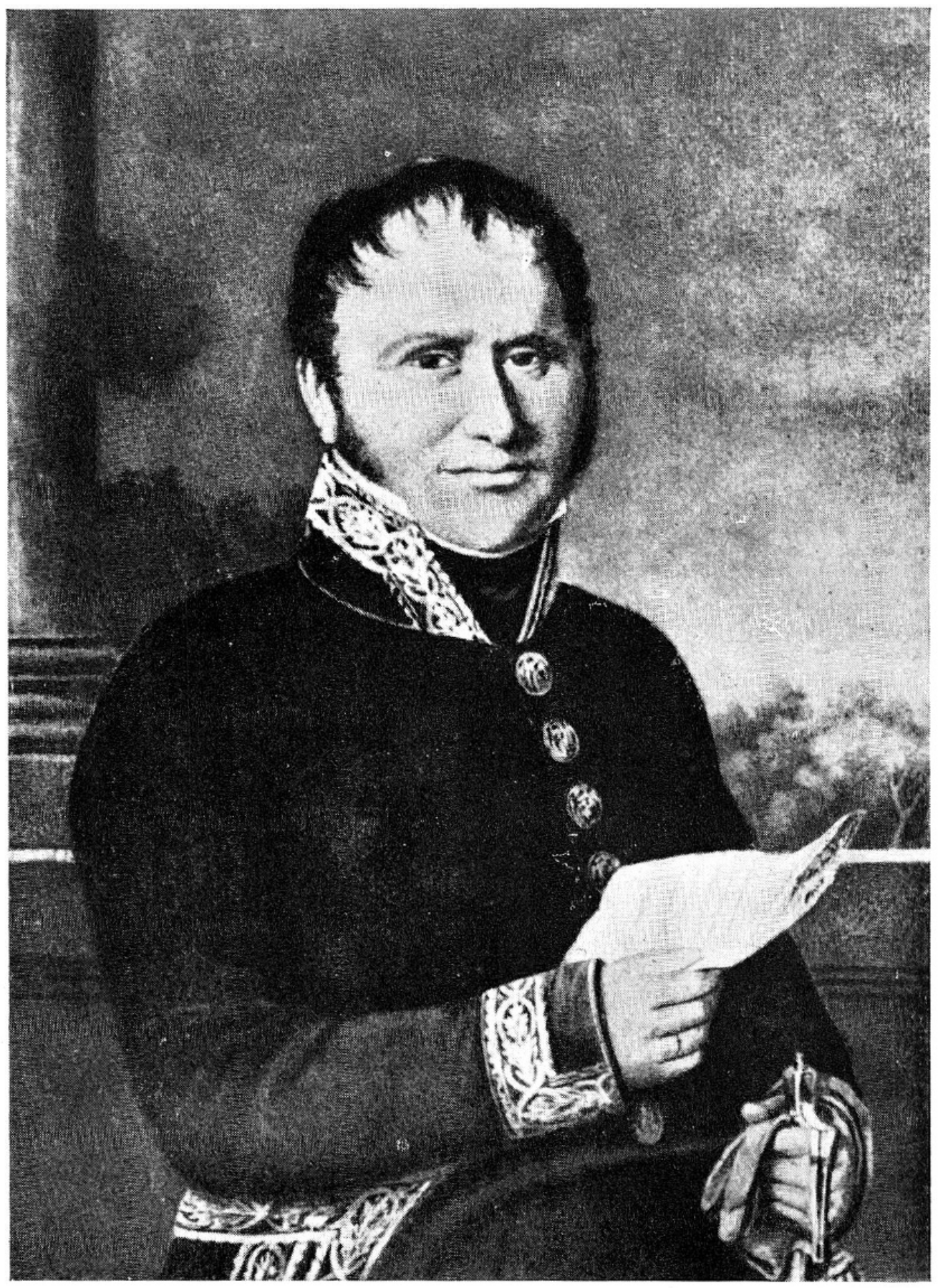

Fig. 1. Tommaso Rima (1775-1843) in uniforme di chirurgo militare 


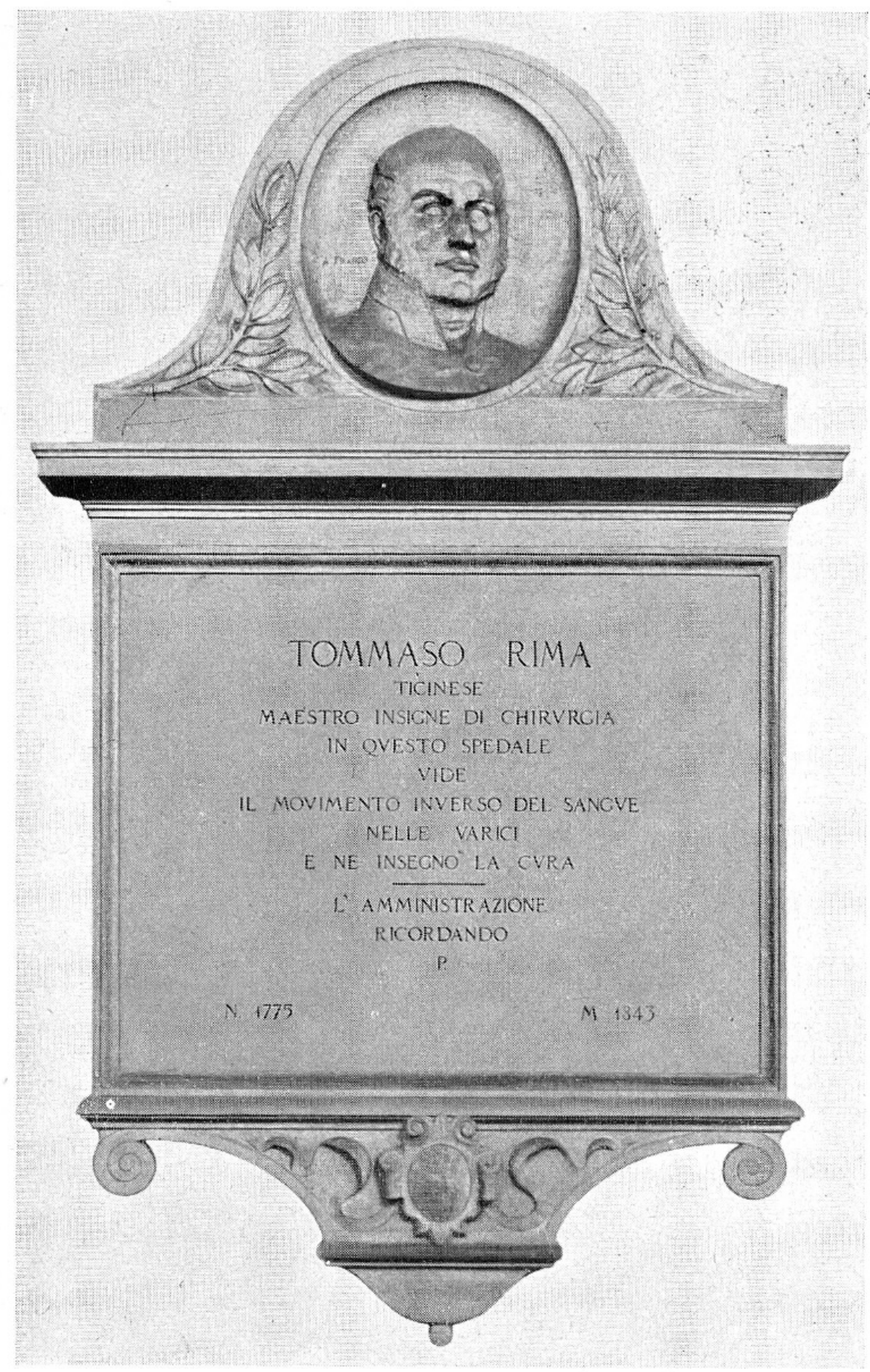

Fig. 2. Ricordo marmoreo di T. Rivi all'Ospedale civile di Venezia, scoperto il 13 settembre $1925^{5}$ (Foto Giacomelli, Venezia) 


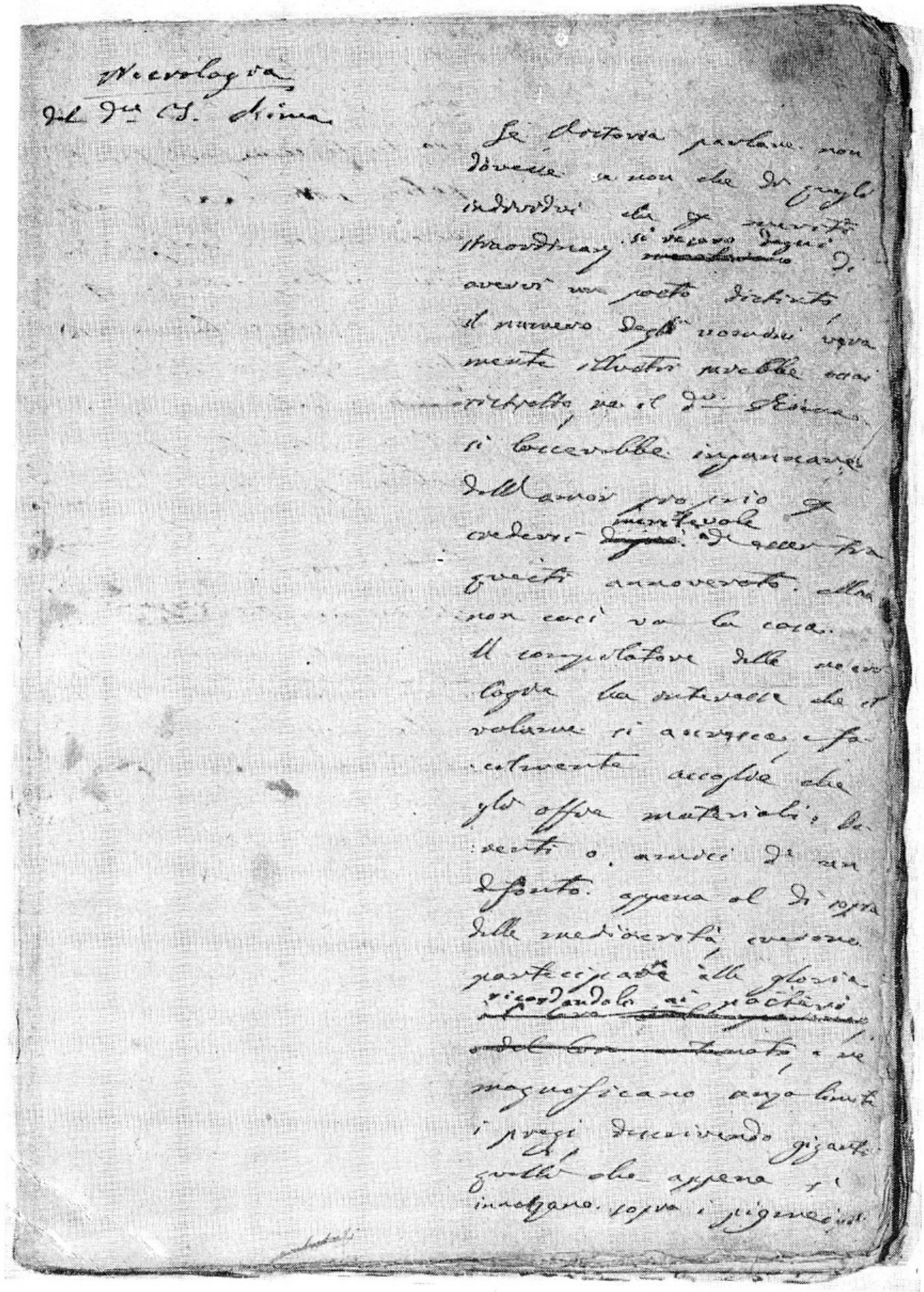

Fig. 3. Prima carta del manoscritto autografo della Necrologia del D. ${ }^{r}$ T. Rima, donato da D. Giondano alla Biblioteca Cantonale di Lugano il 20 febbraio 1947 
Rima D. ${ }^{r}$ Tommaso Antonio, tutt'ora vivente ${ }^{12}$, ne 11811 veniva posto nell'Elenco degli uomini illustri della sua patria [vedi Dizionario Storico Ragionato degli uomini illustri del Cantone Ticino del Padre Lettore Gio. Alfonso Oldelli di Mendrisio, Lugano 1807, continuazione in altro volume, Lugano 1811, pag. 61] ${ }^{13}$.

Li 11 dicembre 1775 nasceva pronipote dell'altro Tommaso già citato a pag. $153^{14}$. La famiglia già vertendo alla decadenza, primeggiava però ancora in quella epoca nel picciolo villaggio d'Albairone nella valle d'O(n)sernone distretto di Locarno, Cantone del Ticino nell'Elvezia.

Fanciullo veniva in compagnia d'un suo maggior fratello affidato alla cura del parroco di Solduno, che teneva in pensione ragazzi di varie distinte famiglie di quei dintorni; ma la cui occupazione era piuttosto la caccia che l'istruzione dei fanciulli con immeritata confidenza consegnatigli. Dava in quella casa i primi indizj di buon cuore, e d'amicizia, seppure non lo volessimo chiamare dabbenaggine. Permetteva ad un suo maggior condiscepolo che gli recidesse la più gran parte di capelli, perchè potesse adornarsene con una finta coda.

Prosseguiva i studj grammaticali nelle pubbliche scuole di Locarno. Ma non si fu che in quelle del collegio de' Chierici Regolari Somaschi di Lugano che potè seguire un metodo di regolare istruzione, incominciando quei studj che aveva diritto di credere percorsi con distinzione precedentemente. Vestito l'abito chiericale sembrava destinato alla carriera ecclesiastica, avendo anche recitato tre orazioni panegiriche. Ma non era quello lo stato a cui si sentiva chiamato.

Compita la rettorica, veniva nel 1793 inviato nella città di Roma a studiar filosofia in quel rinomato Archiginnasio della Sapienza. Ivi si applicava nello stesso tempo, e progressivamente in 6 anni che vi dimorò all'anatomia, alla Fisiologia, alla medicina ed alla chirurgia, ed alle altre scienze che sono con esse in rapporto. In merito di pubblici esami otteneva posto nei quattro grandi Archiospitali $^{15}$ di quella metropoli.

[Per i giovani aspiranti di medicina, e di chirurgia che amano di fare il corso pratico-teorico in uno di quei grandiosi stabilimenti, viene aperto un pubblico concorso. Vi sono ammessi mediante esame verbale (dopo verificati i studj preliminari) sotto la presidenza della superiore Deputazione. Secondo il giudizio dei medici, e chirurghi Primarj esaminatori, vengono classificati in una serie progressiva gli ammissibili. In proporzione che i medici e chirurghi praticanti abbandonano l'ospitale, vengono chiamati i nuovi ammessi a riempirne i vuoti. Ivi progrediscono da prima in ragione di anzianità, in sola via provvisoria. Vacando posti nella gerarchia chirurgica vi vengono promossi gli alunni interni in forza di esami di confronto sino ad occupare il maggiore, cioè di medico assistente e di chirurgo sostituto. Sussidiano in tal qualità giornalmente i Primarj, e ne fanno le veci in mancanza. In tal carico possono continuare sino ad un triennio. Cotesto titolo onorifico è molto valutato nei concorsi pubblici. Esso dà diritto esclusivo al Primariato, che si conferisce però per elezione e non per esame.] 


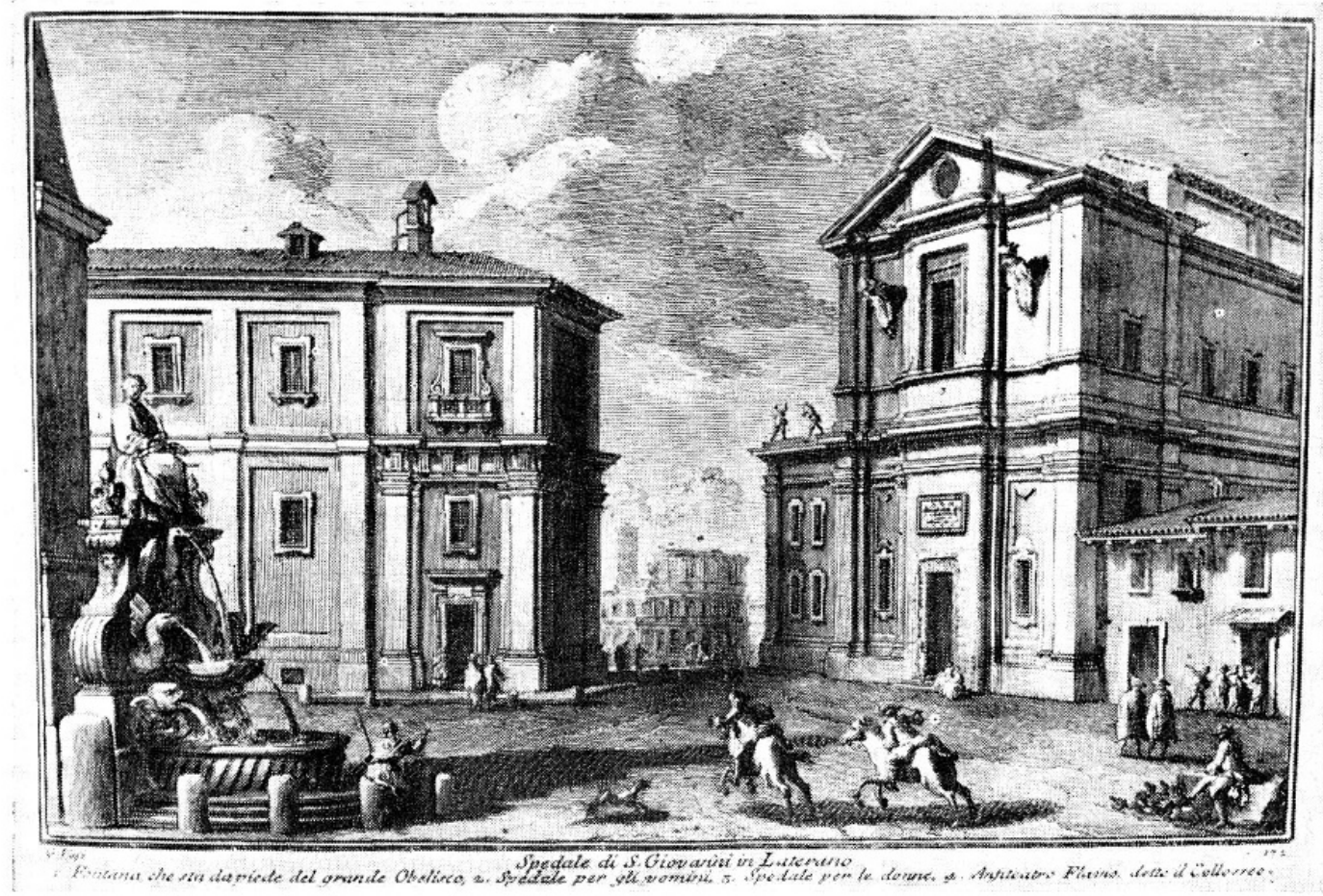

Fig. 4. L'Arcispedale romano del Ss. Salvatore in S. Giovanni al Laterano, dove T. Rima iniziò la sua carriera di chirurgo. Incisione, da G. VASI, Delle magnificenze di Roma antica e moderna. Libro nono che contiene $i$ colleg $j$, spcdali, e luoghi pii . . Roma 1759, tav. CLXXII

Ma preferiva il Rima quello di S. Giovanni in Laterano per la pratica cui doveva applicarsi, siccome quello in cui vi aveva già posto il suo maggior fratello, studente di medicina.

[Instradato in quell'ospitale vi si trattenne per riflessi parziali. Non era il luogo ove per istituto si curassero le malattie chirurgiche. In sei anni di stabile permanenza confessava il D. ${ }^{\mathrm{r}}$ Rima di non avervi veduto che tre sole tra le grandi operazioni. Furono queste una demolizione di mammella per scirro: una amputazione di gamba fatta col segare semplicemente le ossa, giacché la porzione di gamba cangrenata si era lasciata separare spontaneamente con lo sfacelo. Fu la terza praticata dal chirurgo sostituto suo antecessore, infelicemente per ernia carcerata.]

Compiva egli rapidamente la sua carriera; giacché in soli tre esami di confronto avvanzava sopra tutti i competitori della sua classe, e giungeva al posto di chirurgo sostituto. Occupava a quell'epoca quello di Primario Tommaso Maria Celoni ${ }^{16}$ uomo noto per più produzioni estranee alla professione, nella reppubblica letteraria. Il suo impiego nell'ospitale era conciliabile con la frequenza alle lezioni che si davano nell'Archiginnasio della Sapienza ove dettava chirurgia il Professor Sisco ${ }^{17}$ di nazione Corso, che vecchjssimo conservava cordiale memoria 
del suo alunno, e lo rammentava spesso nelle sue lezioni alla Scolaresca Romana, particolarmente trattando delle varici. Laureato già in medicina vi otteneva nel pubblico concorso del 1798 il dottorato ad honorem in chirurgia.

[Alla fine d'ogni anno scolastico è antico uso dell'Università di Roma per incoraggiare i giovani a distinguersi, di premiare quello che in ciascuna facoltà si è eminentemente distinto sopra i suoi condiscepoli. Sono ammessi al cimento dell'esame in pubblico tutti gli aspiranti che hanno terminato il corso. Uno solo è il premiato. A questo si conferisce con particolare apparato il distinto diploma di medicina ... di chirurgia ... di legge ... secondo il ramo della facoltà cui ha aspirato. È esente da qualunque dispendio, restando al premiato un pubblico attestato in quel diploma, che gli accorda preferenza a parità di merito nei successivi aspiri. Un documento qualunque è più o meno valutabile secondo la fonte da cui emana. Prima che le università fossero radicalmente sistemate, in alcune di esse era facile carpire il diploma di chirurgia. Per questi abusi, si meravigliano ancora le popolazioni di veder tra gli esercenti la chirurgia alcuni avvanzi del secolo passato, gravi d'anni e d'ignoranza incorrere negli errori i più grossolani, incapaci, non dicasi, di stendere una perizia ragionata sopra argomento che puole decidere della vita, e dell'onore di un disgraziato; ma inetti a scrivere un periodo in cui vi sia buon senso e concordanza. Non era, almeno nell'epoca di cui trattasi, la chirurgia a miglior condizione in Roma. Poco era sufficiente per esser matricolato chirurgo. Bastava un esame presso il Protomedico. Copriva allora questo carico onorifico l'Ecc. ${ }^{\mathrm{mo}}$ Sig. ${ }^{\mathrm{r}}$ Dott. ${ }^{\mathrm{e}}$ B. ${ }^{18}$ Piemontese. Doveva licenziarsi in chirurgia il Giovane Giuseppe De R . . Romano, che sebbene abbastanza istruito, era troppo timido per render conto di sè in un esame. Altro suo compagno fu officiato a presentarsi in sua vece. Sostenne il sostituto col nome del De R . . un breve esame nella casa del Protomedico; ed il diploma fu rilasciato al De R. Si aggiungeva la circostanza favorevole che il vecchio Protomedico era incomodato da leggiera ottalmite quasi abituale e riceveva in una stanza appena rischiarata di una fosca luce. $\grave{E}$ un fatto di cui il D. ${ }^{\mathrm{r}}$ Rima ne accertava la verità; ed egli non era tale da mentire.]

Durante il triennio in cui il D. ${ }^{\mathrm{r}}$ Rima occupava la carica di sostituto veniva eletto chirurgo della città di Albano. Vi esercitò per due mesi nel 1798 obbligato dalle convenienze; ma vi rinunciava per compiere il corso triennale di sostituto nell'ospitale di Roma, da cui si era assentato con permesso.

Nel generale sovvertimento d'Italia sulle disgraziate ruine del Governo Pontificio si pretese erigersi una così detta Repubblica Romana. Una milizia si era arruolata sotto le bandiere tricolorate divisa in varie legioni. Alla prima di queste veniva chiamato dal superior comando il D. ${ }^{\mathrm{r}}$ Rima per chirurgo Maggior del Reggimento, ed accettava il 1799 incominciando la sua carriera militare col prestare l'opera sua salutare nelle due campagne che ha fatto quel corpo nei dipartimenti allora nominati del Cimino e del Clitunno. Il rovescio sofferto dalle 
falangi repubblicane nell'alta Italia fu il preludio di quello che per concomitanza doveva accadere nella sua parte meridionale. Nella dissoluzione dei soldati Italiani, il Rima sebbene avesse indossato la divisa senza esservi spinto da spirito di partito, si manteneva qual suole sempre uno Svizzero fedele alle sue bandiere, e le seguiva senza esitanza in Francia ove dovevano esser trasportate le truppe in forza di una capitolazione con la flotta Britannica.

[Nel tragitto che faceva da Civitavecchia a Marsiglia sotto la scorta Inglese giungeva felicemente avanti Bastia. Una lancia della sanità marittima comunicava al capitano della nave che Napoleone Bonaparte reduce in maniera portentosa dall'Egitto era sbarcato il giono inanzi in Corsica, e che si trovava in quel punto in Ajaccio sua patria, disposto a prosseguire prontamente il suo viaggio per Francia.]

Gli avvanzj delle poche legioni Romane ebbero il primo soggiorno nel loro camino in Avignone che richiamava loro l'antica residenza dei Pontefici Romani, e la culla di quella donna che tanto rese celebre la tromba del Petrarca. Furono diretti nella Vandea, ma la pacificazione già seguita rendeva inutile l'ulteriore destinazione di armati.

[Un favorevole azzardo portò il Rima ad alloggiare di passaggio in casa di

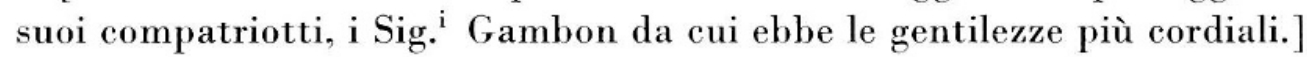

Furono perciò riuniti in una Legione Italica in Digione che formando parte dell'armata di osservazione, rammassata quasi per incanto dal primo console, veniva nel giugno del 1800 a riconquistare l'Italia sulle pianure di Marengo. Sebbene al seguito dell'esercito, giungeva nonostante in tempo di rendere utili servigi in Allessandria ai feriti della memoranda battaglia. Tra quelli gli si porgeva la triste occasione di chiuder gli occhj ad un nobile suo concittadino e condiscepolo, che militando sotto le opposte bandiere spirava tra le vittime di quella micidiale disfatta.

Mentre sostavano l'armate intente ad occupare la linea del Mincio stabilita dall'armistizio, egli rivedeva per giorni la patria che sebbene neutrale era stata con la Lombardia invasa dalle truppe Francesi discese dal San Gottardo for̈mando l'ala sinistra dell'esercito.

S'allestiva intanto la spedizione d'un corpo d'armata ad invadere la Toscana. Egli era per ciò chiamato al Quartier Gle. di Bologna nell'ottobre del 1800 per seguire la divisione del General Pino ${ }^{19}$. Varcati con essa gli Appennini, gli veniva affidato il servizio superiore per vari mesi degli ospitali di Pistoja, di Lucca e successivamente di Siena. Sgombravano quel gran ducato le truppe Reppubblicane per cedere il luogo a quelle del nuovo Re d'Etruria riconosciuto nella pace di Lunéville.

Terminavano così l'incombenze del Rima per cui restituivasi sul finire del 1801 in Lombardia; e come tutti gli altri chirurghi dell'armata si sottometteva in Milano a rigoroso esame per riconoscersi chi meritasse realmente di conservare il grado 
di cui indossavano la divisa. $\grave{E}$ noto che in momenti di confusione s'introducono tra gli ufficiali di salute come nelle armi facoltative individui che non sempre hanno l'istruzione necessaria per conservarvisi con onore. Con vivo applauso, e somma lode degli Esaminatori veniva riconosciuto il D. ${ }^{\mathrm{r}}$ Rima meritevole del rango che occupava tra $\mathrm{i}$ chirurghi maggiori dell'armata Italiana, e veniva nel 1802 attivato nel grande ospitale di Sant'Ambrogio in un grado secondario provvisoriamente sino a che potesse esserlo nel rango che gli si competeva.

Le dissensioni insorte allora tra i membri componenti il consiglio di sanità militare costrinsero il Governo allora presieduto a nome di Napoleone Bonaparte dal vice-Presidente Melzi, a sciogliere il consiglio. Gli attributi dei tre ispettori di sanità militare vennero concentrati col nome di Direttore ${ }^{20}$ nell'integerrima persona del D. ${ }^{r}$ Giacomo Rezia ${ }^{21}$, Professore Emerito della Università di Pavia. A verificare l'operato della precedente commissione di esame, altra ne veniva nominata dal Governo, e sotto altra norma tracciata dal nuovo direttore della sanità militare. Il D. ${ }^{\mathrm{r}}$ Rima che ultimo aveva subito l'esperimento sotto la cessata e trovandosi in Milano fu il primo chiamato a render conto delle sue cognizioni teorico-pratiche sotto la nuova. Più brillante e clamoroso se fosse stato possibile ne emergeva il giudizio, per cui con vera esultanza del nuovo suo superiore, veniva dal Governo con speciale decreto del 2 aprile 1803 confermato nel suo grado di chirurgo Maggiore nell'armata Italiana. Conseguenza di tale conferma governativa veniva egli nel mese stesso di aprile destinato a chirurgo Primario dell'ospitale militare alla destra del Po in Modena, non che della Scuola militare del genio, e dell'artiglieria ivi parimenti stanziata.

Contemporaneamente a questi impieghi stabili riceveva egli altri importanti ed onorevoli commissioni dal Ministero della Guerra. Nel 1804 fu istituita la prima leva di coscritti. A dirigerla, e sorvegliarla furono nominate due commissioni col nome di Giuri centrale, di cui uno risiedeva in Milano per i dipartimenti alla sinistra del Po; l'altro in Bologna per quelli alla destra. Era ciascun composto di un Generale Presidente, d'un Ispettore alle rassegne, e d'un ufficiale maggiore di sanità. Il Rima veniva destinato a quello di Bologna, e vi compiva le sue incombenze con piena soddisfazione del Ministero come al dispaccio relativo del ${ }^{22}$

Alla superiore autorità amministrativa era portata qualche lagnanza dai militari curati negli ospitali civili della Romagna. Il D. ${ }^{r}$ Rima vi veniva spedito nel maggio del 1805 a farne l'ispezione, a verificarne le lagnanze, ed a farvi diritto se ragionevoli. Egli vi riesciva in modo d'accontentare i reclamanti concertando i provvedimenti con le presidenze civili senza ledere le loro convenienze.

Nell'anno stesso due Reggimenti Francesi si chiamavano colla previdenza del Regnante dalla Corsica a rinforzare la linea dell'armata ch'era già forse destinata ad agire sull'Adige. Essi approdavano a Livorno ove infieriva la febbre gialla ${ }^{23}$. Il cordone sanitario divideva la Toscana ai confini del Regno d'Italia. Era decretato che la truppa, isolata venisse a pernottare sull'Appennino; e che dall'ac- 
campamento si trasportasse direttamente al Forte Urbano vicino al Panaro per scontarvi la contumacia, e subirvi la disinfettazione imposta dalle prescrizioni sanitarie. Il D. ${ }^{\mathrm{r}}$ Rima era destinato qual commissario del cordone a dettarne le discipline, ed a sorvegliarne l'esecuzione.Egli seppe condursi in modo da impedire ogni propagazione di contagio di conciliarsi la benevolenza dei militari, e di ben meritare del governo e del Sovrano.

Si sopprimeva intanto l'ospitale militare centrale in Modena. Il Maresciallo Massena concentrava l'armata Francese sull'Adige; e rompendo la guerra, forzava il passaggio di quel fiume. Il D. ${ }^{\mathrm{r}}$ Rima veniva destinato a quella spedizione e si assegnava in qualità di chirurgo Maggiore nel Reggimento Dragoni della Regina, che l'accoglieva con dimostrazioni della meritata stima, e con cordiale deferenza.

Superata la linea dell'Adige quella campagna non offrì che poco lavoro ai chirurghi d'armata. Una ritirata comendevolissima si compiva da S.A. l'Arciduca Carlo. L'armata Francese estendeva la sua vangुaardia sino alla Sava. Il D. ${ }^{r}$ Rima

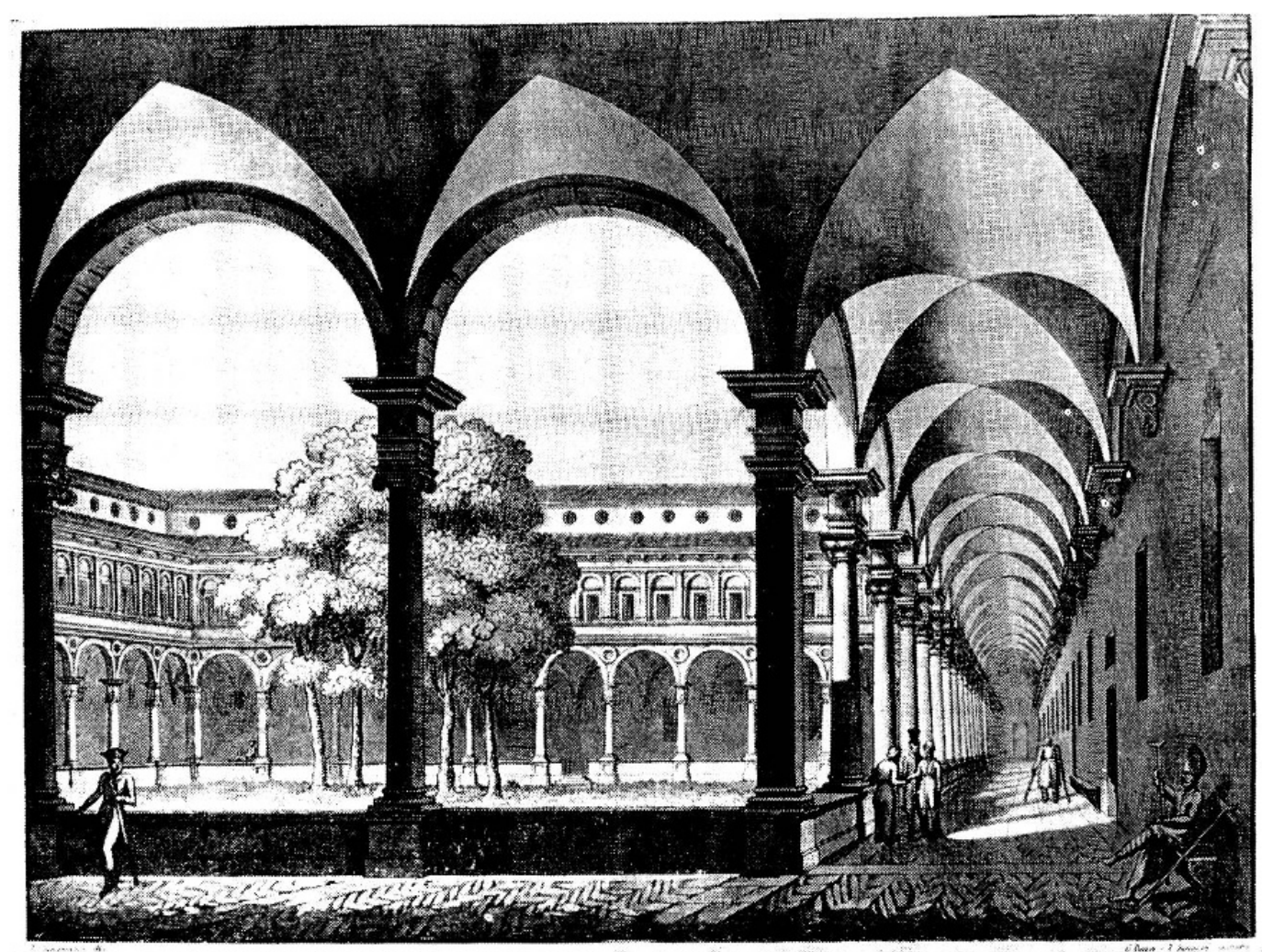

Fig. 5. L'Ospedale militare di Milano, situato nei locali dell'ex-monastero cisterciense di S. Ambrogio, celebre pei saoi chiostri bramanteschi e oggi sede dell'Università Cattolica del Sacro Cuore. Incisione di G. Bussi e V. Raineri da disegno di A. Sanquirico; da G. Ferrario, Monumenti sacri e profani dell'Imperiale e Reale Basilica di Sant'Ambrogio in Milano, Milano 1824, tav. XXX a p. 208 
seguendo il Quartier Generale della Divisione Ser(r)as ${ }^{24}$, veniva destinato a dirigere il servizio dell'ospitale di Trieste, ove si erano concentrati gli ammalati per il maggior numero dell'armata Imperiale Austriaca.

Si convenne la pace col trattato di Vienna ${ }^{25}$. L'armata si ritirava dietro l' Isonzo, ed una porzione di questa volgevasi al mezzo giorno d'Italia capitanata dallo istesso maresciallo Massena alla conquista di Napoli. Il D. ${ }^{r}$ Rima seguiva quell'armata, e dopo occupato quel soglio dal Principe Giuseppe Bonaparte, che ne veniva dichiarato Re, ritornava nell'estate del 1806, col Reggimento ad occupare il porto di Ancona.

Da quella piazza veniva egli richiamato dal Governo ad occupare il posto di Primario chirurgo dell'ospitale di Milano. Il suo Reggimento era dispiacente di vederlo partire, ma rendeva giustizia a suoi meriti per esser chiamato alla capitale, molto più da che quel corpo armato cessava d'esser in faccia al nemico. Dal 1806 al 1811 continuava il servizio superior di chirurgia nell'ospitale suddetto. Varie altre incombenze si addossavano al Rima o inerenti a quel posto, o per commissione personale. Era tra le ordinarie quella di comporre col medico Primario ch'era allora il D. ${ }^{\mathrm{r}}$ Cocchetti ${ }^{26}$, e coll'Ispettore Generale di sanità ch'era l'emerito Prof. Rezia ${ }^{21}$, di comporre dico una commissione speciale per giudicare in revisione sulla idoneità dei coscritti che per le loro possidenze appartenevano ai corpi della Guardia Reale, e che dalle rispettive Prefetture fossero stati giadicati inabili al servizio militare.

Per ordine speciale di S.E. il ministro della guerra veniva spedito ad ispezionare l'ospitale di Cremona; ed associato al D. 'Bussan (?) riceveva l'incarico d'ispezionare il corpo d'artiglieria stanziato in Pavia per estrarne gl'invalidi, o veterani resi inetti al servizio attivo.

[Fu in quella circostanza che il Prof. Cairoli27 le mostrava ormai convalescente il primo suo operato di varice alle gambe col metodo di Home ${ }^{28}$, cioè con l'allacciatura della gran safena in vicinanza dell'articolazione del ginocchio.]

Con speciale decreto di S.A.I. il Principe Vice Re del . . $1807^{29}$ le veniva conferita la cittadinanza Italiana, e le veniva annunciato con soddisfazione dal dispaccio di S.E. il Ministro dell'Interno in data del ${ }^{30}$

Con sovrana determinazione veniva stabilito nell'anno stesso che vi fosse nel Regno un chirurgo in capo [il grado di chirurgo in capo corrispondeva a quello di Colon(n)ello; v. legge 11 decembre 1806] degli ospitali militari Italiani, e questa onorifica carica veniva al D. ${ }^{\mathrm{r}}$ Rima conferita il 10 settembre dell'anno suddetto, conservando nel medesimo tempo il carico di Primario chirurgo dell'ospitale militare nella capitale del Regno.

Esisteva sino a quell'epoca una commissione medico-chirurgica di Esaminatori civili alla quale era rimesso il giudizio sulla idoneità dei chirurghi militari. Dipendeva da questa il riconoscere quai potessero esser ammessi nell'armata, quali meritassero d'esser avvanzati in grado. Da quell'epoca la commissione fu mista 
di Professori civili e militari ed il D. ${ }^{\mathrm{r}}$ Rima ne diveniva membro stabile nella sua qualità di chirurgo in capo il 17 agosto 1808.

[Il metodo di quell'esame farà sempre onore alla giustizia, ed alla perspicacia di chi l'ha progettato: il Cav. Rezia ${ }^{21}$ allora Ispettore Generale della sanità militare. Egli non si era riserbato che la presidenza; rinunciando al voto. Quattro erano gli esperimenti cui venivano sottoposti gli aspiranti, e divisi in altrettante giornate. Consisteva l'esame della prima in varie interrogazioni verbali che da sei esaminatori si facevano in seduta per oltre un'ora cumulativamente al candidato. Ciascun esaminatore scriveva nel registro quel grado di soddisfazione a cui aveva corrisposto in ogni giorno. Era il secondo destinato allo scioglimento di un caso pratico in iscritto per il quale si accordava il tempo necessario in una camera custodita. Si compiva il terzo esperimento al letto di un ammalato che veniva sommesso all'ispezione ed alle interrogazioni del candidato. Da questo doveva emergere la diagnosi, la prognosi e proposizione di cura accompagnata da qualche raggionamento sulla malattia stessa. Chiudeva finalmente l'ultimo esame una operazione sul cadavere. Dai voti degli esaminatori in queste quattro giornate risultava il giudizio sul merito relativo; ed il fatto fece prova che chiunque fu ammesso per tal mezzo al servizio sanitario dell'armata, si mostrò uomo di merito reale e meritevole di distinzioni. Componevasi la commissione:

$I^{0}$ dall'Ispettore Generale di sanità militare Cav. Rezia ${ }^{21}$ Presidente dai Professori

\begin{tabular}{l|l}
$\begin{array}{l}\text { Paletta }^{31} \\
\text { Monteggia }^{32}\end{array}$ & civili \\
$\begin{array}{l}\text { Rasori } \\
\text { Assalini }\end{array}$ & clinici misti \\
$\begin{array}{l}\text { Cocchetti } \\
\text { Rima }\end{array}$ & Primarj militari.]
\end{tabular}

Partiva per la Spagna nel 1808 il Professor Assalini ${ }^{31}$. Perché non mancasse nella di lui assenza quel ramo di chirurgico insegnamento, si aggiungeva al chirurgo in capo D. ${ }^{\mathrm{r}}$ Rima, oltre le sue incombenze, l'onorevole incarico di Professore di clinica ed operazioni chirurgiche. La straordinaria commissione veniva disimpegnata con speciale soddisfazione del Ministero e del R. Governo che con repplicati dispacci si degnavano partecipare al Professore. Tale pratico esercizio veniva coronato dal profitto dei chirurghi dell'armata che per tale oggetto venivano per turno mandati alla capitale, non che da numerosi concorrenti anche tra i civili cui era accordato l'accesso. Non era difficile al Professor Rima di esercitare i giovani alunni nelle operazioni chirurgiche che spesso aveva avuto occasione di mostrare loro sul vivente, tanto sui campi di battaglia, quanto nei varj ospitali, Ma ciò che merita eloggio speciale si fu d'aver saputo combinare senza esaltarsi 
la patologia e terapeutica chirurgica nelle sue lezioni, da non essere in opposizione coi principi della teoria del contro-stimolo che contemporaneamente si dettavano dal clinico medico suo collega nell'istesso stabilimento, il celebre Prof. Rasori ${ }^{33}$.

Mancava nel 1809 il chirurgo in capo dell'armata Francese al Quartier Generale di Milano, nè a tutto bastava la presenza del medico in capo. Il Prof. Rima veniva associato a questi per le girate d'ufficio ad assistere il capo di stato maggior generale per togliere dall'armata attiva i militari Francesi che n'erano divenuti inabili, e per dare i suoi consiglj per tutto ciò ch'era di chirurgica pertinenza e negli ospitali, e nei Reggimenti.

L'utilità riconosciuta della clinica chirurgica di Milano, determinò il R. Governo Italiano a renderla comune oltre a quello della Marina in Venezia anche ai due grandi ospitali di Mantova e di Ancona concentrando le doppie incombenze nella stessa persona del Primario chirurgo. Doveva riunirsi nel Cav. Assalini ${ }^{34}$ la duplice carica nella capitale, giacché essendo contemporaneamente chirurgo di S.A.I. il Principe Vice-Re non doveva abbandonare la residenza del Principe.

Veniva per ciò il D. ${ }^{\mathrm{r}}$ Rima traslocato con i due attributi al grande ospitale militare di Mantova col primo giorno del 1811. Lo preveniva in quella città la fama di valente operatore che si era già acquistato sui campi di battaglia e nei varj ospitali d'Italia. Si rinnovavano i fatti luminosi in quella città; ed a rammentarne uno S.A. il Principe Luigi Gonzaga otteneva dal ferro chirurgico per opera del Rima quella guarigione radicale che implorò invano per due anni in Parigi, e che non aveva ottenuta che incompleta dal valente Monteggia ${ }^{32}$ in Milano $^{35}$.

$\mathrm{Fu}$ in quell'ospitale militare che avendo l'opportunità d'avere ammalati varj dai soldati veterani ch'ivi avevano stanza, faceva i primi esperimenti sopra la cura radicale delle varici alle gambe ${ }^{36}$. Il Pavese Prof. Volpi ${ }^{37}$ li pubblicava nell' $8^{\circ}$ Tomo della sua Traduzione di Richter ${ }^{38}$.

Chiamava al servizio dello stabilimento un suo valente collega il D. ${ }^{\mathrm{r}}$ Solera ${ }^{39}$, e l'otteneva dal Ministero della guerra. Per questa nomina, sebbene giustificata dal merito, ebbe a soffrire varie controversie per parte di alcuni tra i chirurghi militari, che in tale scelta sinistramente volevano interpretare una oltraggiosa preferenza sopra di essi. Ne addusse le ragioni, e le sostenne. Il Solera beneficato fu tra i pochi che gli professò costante gratitudine. Egli giustificò la scelta fatta dal Rima con utili servigi resi all'ospitale sotto entrambi i governi supplendo nelle occasioni alla mancanza dei Primarj.

[ I meriti del Solera erano ben noti a suoi concittadini, di cui piangono ancora l'immatura perdita! Egli fu successivamente Professore clinico, e Primario chirurgo di quel civico ospitale.]

Correva l'epoca allora in cui un grande Esercito s'avviava verso il nord dell'Europa sotto gli ordini del gran Capitano a lottare più contro gli avversi elementi che contro le nemiche falangi. Una numerosa divisione Italiana moveva dal Regno a dividerne gli allori o i cipressi. Il Generale che la comandava richie- 
deva che il Rima l'accompagnasse. Ma non l'accordava il Ministro della guerra, nell'imbarazzo in cui diceva di trovarsi nel momento per sostituirlo nel numeroso ospitale di Mantova.

Un bisogno meno eventuale reclamava nelle Marche alcuni mesi dopo l'opera del D. ${ }^{r}$ Rima. Il D. ${ }^{r}$ Solera $^{39}$ era allora già edotto del servizio militare per poter sostenere le sue veci nell'ospitale di Mantova. Dominava fieramente nei militari che formavano la guarnigione di Ancona, e dei contorni una ottalmia contaggiosa ${ }^{40}$, vecchio ed infelice retaggio dei soldati reduci dall'Egitto. E vita, ed occhj vi perdevano in gran numero gl'infelici che ne venivano attaccati. Giudicava necessario il Ministero che un esperto ed intelligente chirurgo si portasse sul luogo per darvi i necessarj provvedimenti. Sotto la data del 14 novembre 1812 vi veniva spedito in posta il Rima. Riconosciuto a non dubbitare il carattere contaggioso della malattia, furono prontamente attivate tutte le misure sanitarie incominciando dall'isolamento degli infetti. Da questo radicale provvedimento ne derivò il numero sempre più minorato dei nuovi attaccati, ed in due mesi circa non si avevano che a curare le tristi conseguenze della vinta malattia.

Fece sorpresa che dopo due anni ${ }^{41}$ sortisse col nome del D. ${ }^{\mathrm{r}}$ Vasani ${ }^{42}$ di Verona una pretesa Storia dell'ottalmia contaggiosa di Ancona. Si appropriavano gli esperimenti fatti sugli animali, non che quei riflessi che emergevano dagli ufficiali rapporti che al cessato Ministero della Guerra si comunicavano dal Professore Rima nominato allora Ispettore di sanità di quel circondario. Commetteva egli al D. ${ }^{r}$ Vasani mandatogli qual medico soprannumerario (essendovi già gli attivi Bussan[?] e Vespa) per dargli qualche occupazione la visita dei convalescenti di ottalmia. Nessun ammalato di prima invasione ebbe a curare, o se pure qualche ricaduto, solo temporariamente ed abusivamente, giacché era in dovere di rimandarlo tosto nella sala ordinaria della cura. L'ottalmia fu sempre negli ospitali militari del Regno d'Italia di esclusiva pertinenza chirurgica. L'ispettore di sanità o li trattava esso medesimo (?) direttamente, o i suoi chirurghi subalterni, tra i quali primeggiava per rango e per cognizioni il Prof. Bongiovanni ${ }^{43}$ morto pochi anni addietro sulla cattedra d'ostetricia nella R. Università di Pavia. Una confutazione aveva preparato il D. ${ }^{r}$ Rima; ma da che si vidde prevenuto dal D. ${ }^{r}$ Omodei $^{44}$, dimise il pensiero di pubblicarla ${ }^{45}$.

[Il libercolo pubblicato col nome del D. ${ }^{\mathrm{r}}$ Vasani era sopra gl'informi suoi materiali, stato scritto nelle prigioni di Mantova da Giovanni Rasori ${ }^{33}$. Ciò che fu curioso si è che il D. ${ }^{r}$ Rima è stato il canale intermediario per cui passò il compenso pecuniario alla figlia. Se non emergeva la verità, risultava(no) in quel libercolo i meriti del recondito scrittore.]

Avanti che terminasse la sua missione, volendo il Ministero della guerra dare al D. ${ }^{r}$ Rima una ricompensa onorifica e lucrosa, lo commissionava Ispettore di sanità militare per il circondario della Quinta divisione [la quinta divisione comprendeva i tre dipartimenti del Tronto, del Metauro, e del Musone; capo luogo 
Ancona], aggiungendovi per di più il dipartimento del Rubicone. Ospitali, caserme, viveri, corpi militari, e tutto ciò ch'era esercito o vi aveva rapporto, era al medesimo subbordinato per quanto spettava al ramo sanità.

Nel memorando rovescio sofferto dalla grande armata Francese del nord, due divisioni di armati Napolitani eccellentemente montate accorrevano dal mezzo giorno d'Italia col nome di amici sul terminare del 1813 ad occupare la piazza d'Ancona, ed il littorale. A sorvegliare l'approvvigionamento d'assedio di quella piazza era nominato un commitato, ed il 9 novembre 1813 il D. ${ }^{\mathrm{r}}$ Rima vi era prescelto membro. Non appena avevano occupati i forti che cessarono d'esser sospetti gli armati Napolitani dichiarandosi alleati ai nemici di Francia, e del periclitante Regno d'Italia ... ! Privata la guarnigione Franco-Italiana dei principali forti, si ritirava sul solo che gli rimanesse la cittadella di Ancona, ed ivi capitolava dopo un mese di blocco, e dopo il bombardamento di un giorno, essendo dominata dalle due alture fortificate: i cappuccini ed il monte Gardetto (= Cardeto). S'arruollavano sotto il vessillo Partenopeo militari d'ogni arma, sedotti dalla lusinghiera promessa d'un Principe che s'illudeva egli stesso ${ }^{46}$.

Fedele il D. ${ }^{\mathrm{r}}$ Rima a suoi impegni, anzi a suoi doveri non abbandonava nell'avversa sorte i vessilli dell'Aquila Reale. Maggior titolo alla considerazione del nuovo sovrano era secondo lui la costanza con cui aveva servito l'antecessore nelle prospere, come nelle disgraziate vicende. Soffriva con tali principj i disagj dell'assedio, ed i pericoli del seguito bombardamento; ed in forza della capitolazione ritornava in Milano a render conto di sè.

Con dispaccio ministeriale 9 marzo 1814 veniva ivi riattivato in qualità d'Ispettore sanitario per la prima divisione militare. Come tale l'11 maggio veniva sotto l'amministrazione R. Austriaca spedito in Pavia col Sig. Magg. ${ }^{\text {re }}$ Forni(?) ad erigere ed organizzare un ospitale per gli ammalati tedeschi. Nella sua qualità di Ispettore rimetteva quindi con l'ospitale della capitale il servizio sanitario all'autorità austriaca, e passava con il corpo amministrativo allo stato di cuiescenza con pensione provvisoria il primo agosto 1814 .

Un triste incarico ebbe a sostenere in quell'epoca, nei giorni di sovvertimento popolare del 20 aprile. Nella seconda notte che succedeva a quella si recava col tribunale a far estrarre di sotterra la nuda salma del conte Prina ${ }^{47}$ già Ministro delle Finanze per eseguirne l'ispezione d'ufficio. Qual orrendo spettacolo! In una infinità di lesioni che lentamente l'uccisero, non ve n'era una che fosse essenzialmente mortale! Vittima disgraziata della ferocia, e della viltà!

Cessando dal militar servizio otteneva gratis dalla R. Università di Pavia il diploma di libera pratica in Medicina e Chirurgia per tutto il vasto impero dell'Austria. Nello stato di temporaria quiescenza si domiciliava in Pavia perché la sua seconda moglie non si disgiungecse dal figlio precedente ${ }^{48}$, che intraprendeva i studj legali in quella R. Università. Legava per tal modo più stretti rapporti coi più distinti Professori, nè si credeva umiliato dopo d'aver dettato dalla catte- 
dra, di confondersi con la scolaresca, e di gustare le lezioni dei dotti suoi colleghi. E sicuramente egli contò tra i più felici mesi quelli gustati in quel placido soggiorno, in quella sede delle scienze, legando(si) specialmente con il Panizza ${ }^{49}$, con il Cairoli $^{27}$, col Moriggia ${ }^{50}$, collo Scarpa ${ }^{51}$, col Borda ${ }^{52}$, col Tamburini ${ }^{53}$, che si pregiavano di annoverarlo tra i loro amici.

Ma non a lungo restava inoperoso. Il General comando Imperiale austriaco lo richiamava all'attività da prima come capo medico nella fortezza di Legnago ${ }^{68}$, indi nel Reggimento Infanteria del Principe Paar. Accettava il servizio per mostrarsi riconoscente alla confidenza di cui le dava prova l'aulico consiglio di guerra; ma conscio non poter per le circostanze di famiglia continuare nella milizia. Si presentava candidato al concorso di tre diverse cattedre nelle $R$. Università austriache in Italia. Ne otteneva vantaggioso rapporto ${ }^{53^{3}}$ della facoltà medico chirurgica, e favorevole proposizione se ne sommetteva a S.M.I.R.

[Senza rinunciare all'aspiro delle cattedre, nell'atto che otteneva il chiesto congedo dal militare servizio, ed in nome e per comando dell' I.R. aulico consiglio di guerra del 30 novembre $1820 \mathrm{n}^{\circ} 5022 \mathrm{~L}$ in data del 6 decembre dichiarava l'I.R. Direzione medica che il D. ${ }^{r}$ Rima fu sempre molto zelante e diligente nella assistenza degli ammalati, si mostrò in possesso delle più distinte e nigliori cognizioni medico-chirurgiche, la sua morale condotta fu sempre prudente ed inreprensibile, per cui con piacere l'I.R. Direzione coglie l'occasione di attestargli col presente la sua soddisfazione per i buoni servizj prestati all'I.R. armata come chirurgo etc. etc.]

Veniva eletto intanto con vistoso stipendio Primario chirurgo dell'ospitale e della città di Ravenna. Egli l'accettava, sempre disposto a rientrare nello Stato, qualora a S.M. fosse piaciuto nominarlo ad una delle cat tedre per cui era proposto. Ma la cattedra fu rimessa al concorso perché alla proposizione sottoposta a S.M. per la sua nomina fu aggiunto che il petente v'aveva rinunciato accettando impiego sotto il governo Pontificio; e ciò le fu noto dalla bocca stessa di S.M.I.R. in Lubiana. Per circa tre anni sostenne in Ravenna il carico di Primario chirurgo, aprendo con erudita prolusione la scuola per le levatrici, prolusione che la sua modestia fece rifiutare alla rappresentanza comunale che la voleva data alle stampe a spese del magistrato.

[Scriveva il Gonfaloniere il 28 agosto $1820 \mathrm{n}^{\circ} 1381$ «La prolusione ch'ella testè à recitato in questa grande sala comunale, ha riscosso tale applauso e per gli ottimi sentimenti di morale e di scienza, e per la squisitezza dell'erudizione e dello stile, che nell'atto in cui interprete dei sentimenti di tutti i miei concittadini glie ne esterno i più distinti e sinceri eloggi mi pregio di prevenirla che questa Magistratura ha divisato di rendere il di lei scritto di pubblica ragione dandolo alle stampe onde non vi sia chi venghi defraudato del piacere e della soddisfazione di leggerlo, e di far applauso alla di lei dottrina, ed all'eccellente di lei sapienza. Prego quindi etc. etc.».] 
Con ripetute operazioni si confermò in Ravenna la fama che s'era già procurato in Lombardia. In quell'ospitale ripeteva le sue osservazioni pratiche sulla cura delle varici alle gambe. Ivi scriveva la sua memoria sul perfezionamento del processo operativo per l'estirpazione dei testicoli ${ }^{54}$. Ivi rispondeva in istampa ${ }^{55}$ ad un imberbe chirurgo che ignaro dei primi elementi della scienza si era eretto a criticare una operazione di Litotomia sebbene confessasse egli stesso che l'operato perfettamente guarito passeggiasse le contrade della città appena compita la terza settimana. Membro della commissione di sanità, ispezionava le Farmacie, e proponeva co'suoi colleghi utili provvedimenti. Da quella commissione s'introduceva per la prima volta nella legazione di Ravenna una tariffa de' medicinali, la quale, senza poterla a(n)nullare, si riprovava dall'orgoglio del Romano medico colleggio; ma veniva poi con l'opportune variazioni per ordine superiore addottata nell'istessa capitale con generale approvazione delle popolazioni.

Perdeva intanto Venezia con universale compianto nella persona del D. ${ }^{\mathrm{r}} \mathrm{De}$ Marchi $^{56}$ il chirurgo in capo del suo grande ospitale Provinciale. A sostituirlo fu aperto il pubblico concorso. A presentarvisi aspirante fu officiato il Rima. Il faceva, e sopra vari concorrenti ne otteneva la nomina dall'Eccelso Imperiale R. Governo sotto la data del 3 maggio 1822.

Con quale rincrescimento accettassero le Autorità tutte di Ravenna nel mese stesso, e con quale lo vedessero deffinitivamente partirne nel successivo mese di agosto, appare specialmente dai brani di lettere che si trovano tra sue carte, e che vengono in calce trascritte.

[A) Sotto la data 12 maggio 1822 le scriveva S. Eccellenza Monsignor Codronchi $^{57}$ Arcivescovo etc. etc. «La sua lettera del 10 corrente quantunque piena di sentimenti graziosi a mio riguardo, non ha lasciato di eccitarmi nell'animo un vivissimo dispiacere per la perdita che mi annuncia prossima della mia Ravenna di un Professore fornito di tanti lumi, ed animato da tanta pratica nella difficile arte medico-chirurgica. A me certo saranno sempre presenti le molte e singolari sue doti e qualità stimabilissime, e le applaudite operazioni in tanto numero e sì felicemente compite in questo ospitale a vantaggio della languente umanità. Piaccia a lei di conservarmi la sua amicizia e di assicurarsi che mi sarà sempre gratissimo di poterle dar prova efficace di quella particolare e distinta considerazione, e meritato attaccamento col quale etc. etc.».

B) Sua Eminenza il Cardinal Rusconi ${ }^{58}$ legato della Provincia chiudeva la sua lettera 18 agosto $\mathrm{n}^{\circ}$ 2484 "Aggradisca almeno che con la presente le dia una sincera testimonianza della molta gratitudine che le si professa per il fervido zelo impiegato in tutte le cure intraprese, per ogni lor felice e glorioso successo, e più ancora per la pietà e generosità usata sempre verso i poveri, lo che forma il più bell'eloggio dell'animo suo sensibile».

C) Il Gonfaloniere della città, sotto il $n^{0} 1265$ scriveva il 20 agosto $1822^{59}$ «... Questa Magistratura che seppe in ogni incontro apprezzare il distinto di lei merito, 


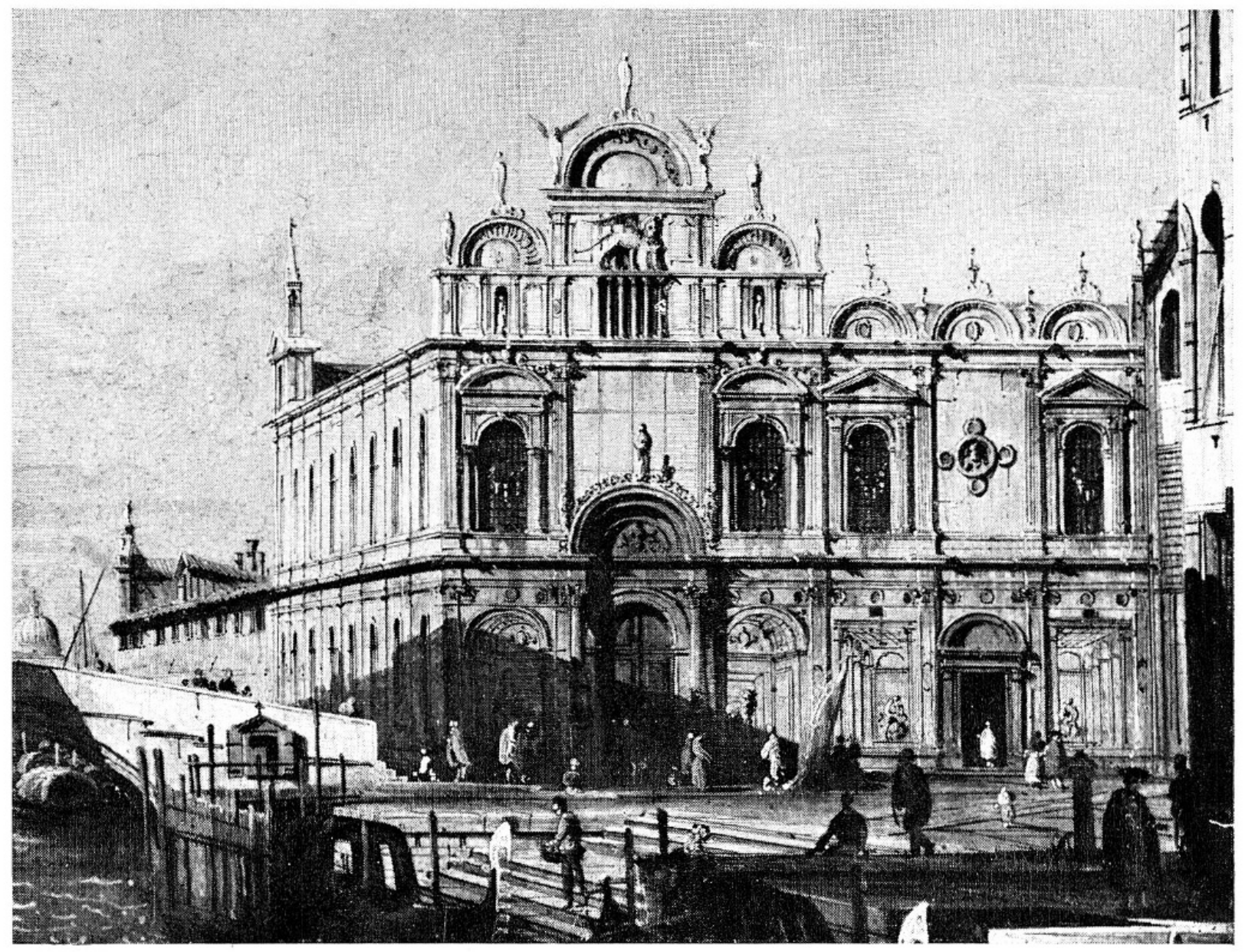

Fig. 6. La Scuola Grande di S. Marco, sede dell'Ospedale civile di Venezia, dove T. Rima, raggiunto l'apice della sua carriera, fiorì come chirurgo primario. Dipinto attribuito a Giovanni Antonio Canal detto il Canaletto (1697-1768). Gallerie dell'Accademia, Venezia

è gravemente sensibile alla di lei perdita, e se può in qualche modo alleviarsi questo sentimento, lo è nel soddisfare ad un atto di giustizia, dichiarandole la piena approvazione, congiunta alla gratitudine pubblica per gl'importanti servigj con assiduità, con somma intelligenza ed umanità, e con successo costantemente felice, resi a chi ebbe ricorso alla di lei assistenza, per la qual cosa ella raccoglie la dolce compiacenza di partire da Ravenna accompagnato dalla stima, e dallo amore universale. Gradisca etc. etc.».

D) La congregazione di carità li 28 agosto 1822 «Ci è dolorosissimo perdere la virtuosa e stimabile di lei persona, la cui esperta mano non cesseranno mai di benedire tante persone infelici curate e portate a salvamento, come la nostra piena soddisfazione per l'esemplarissima condotta sempre mai tenuta, accom- 

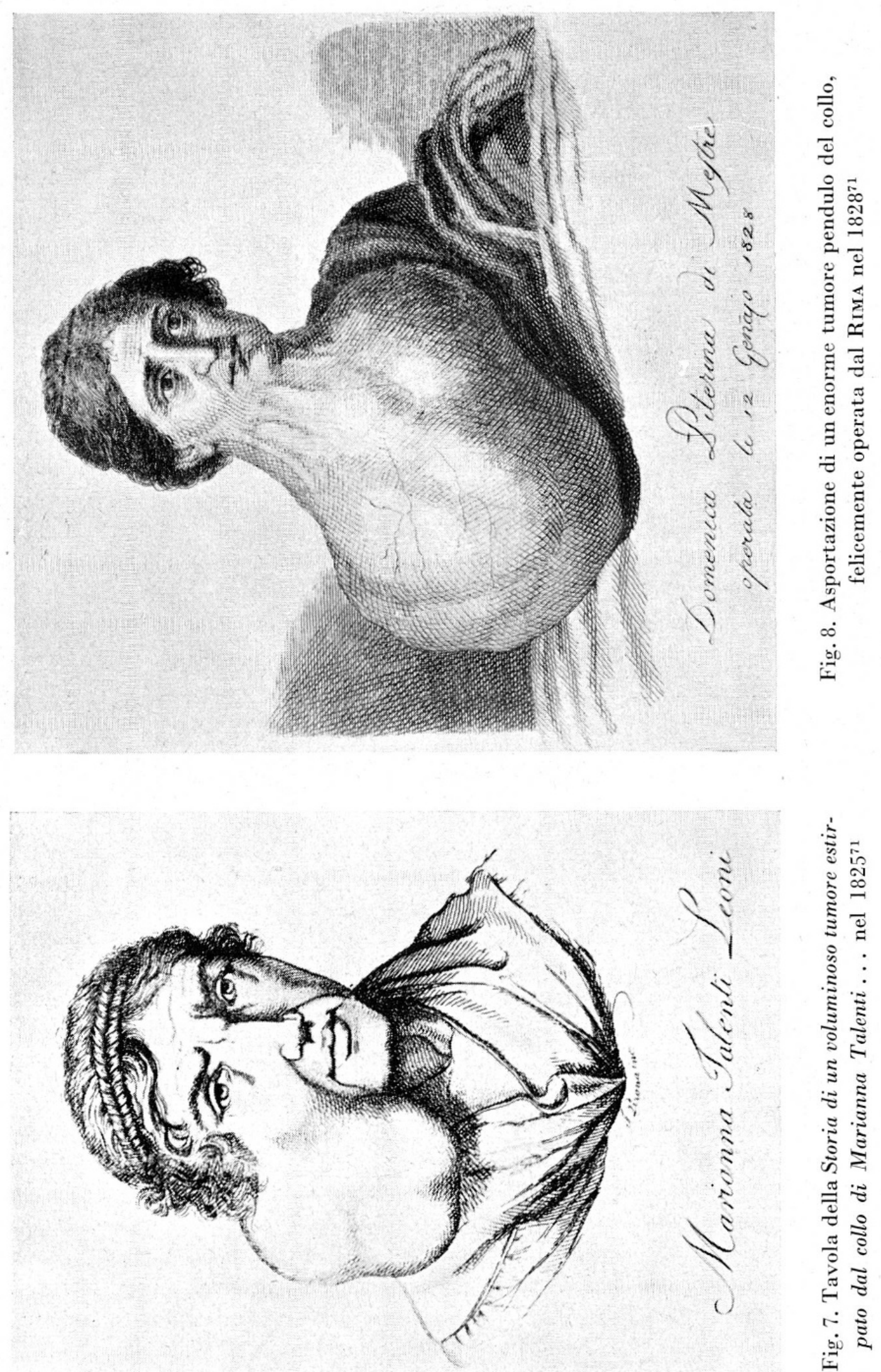


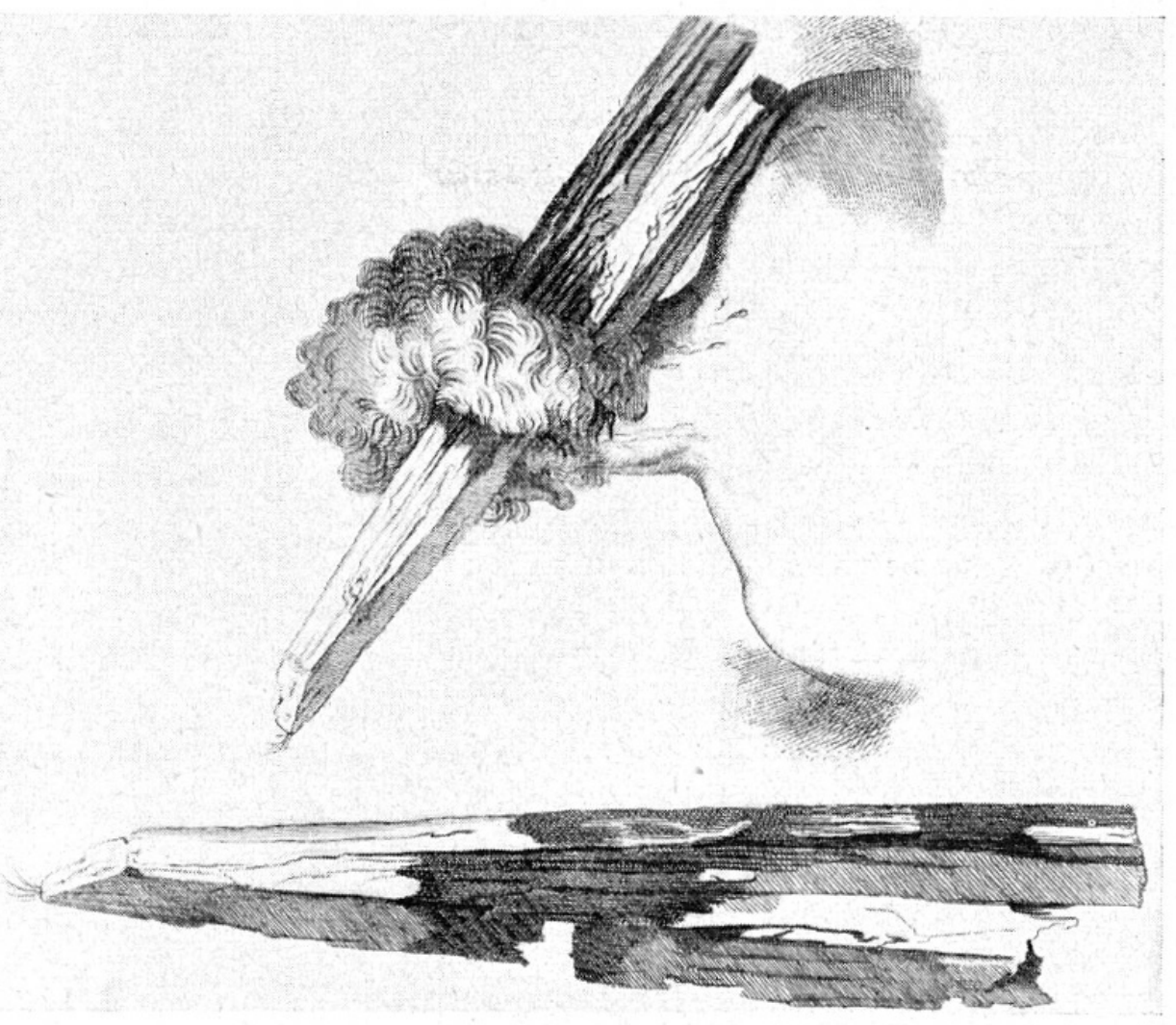

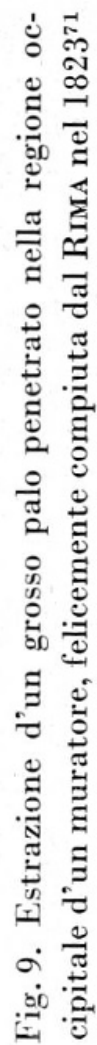

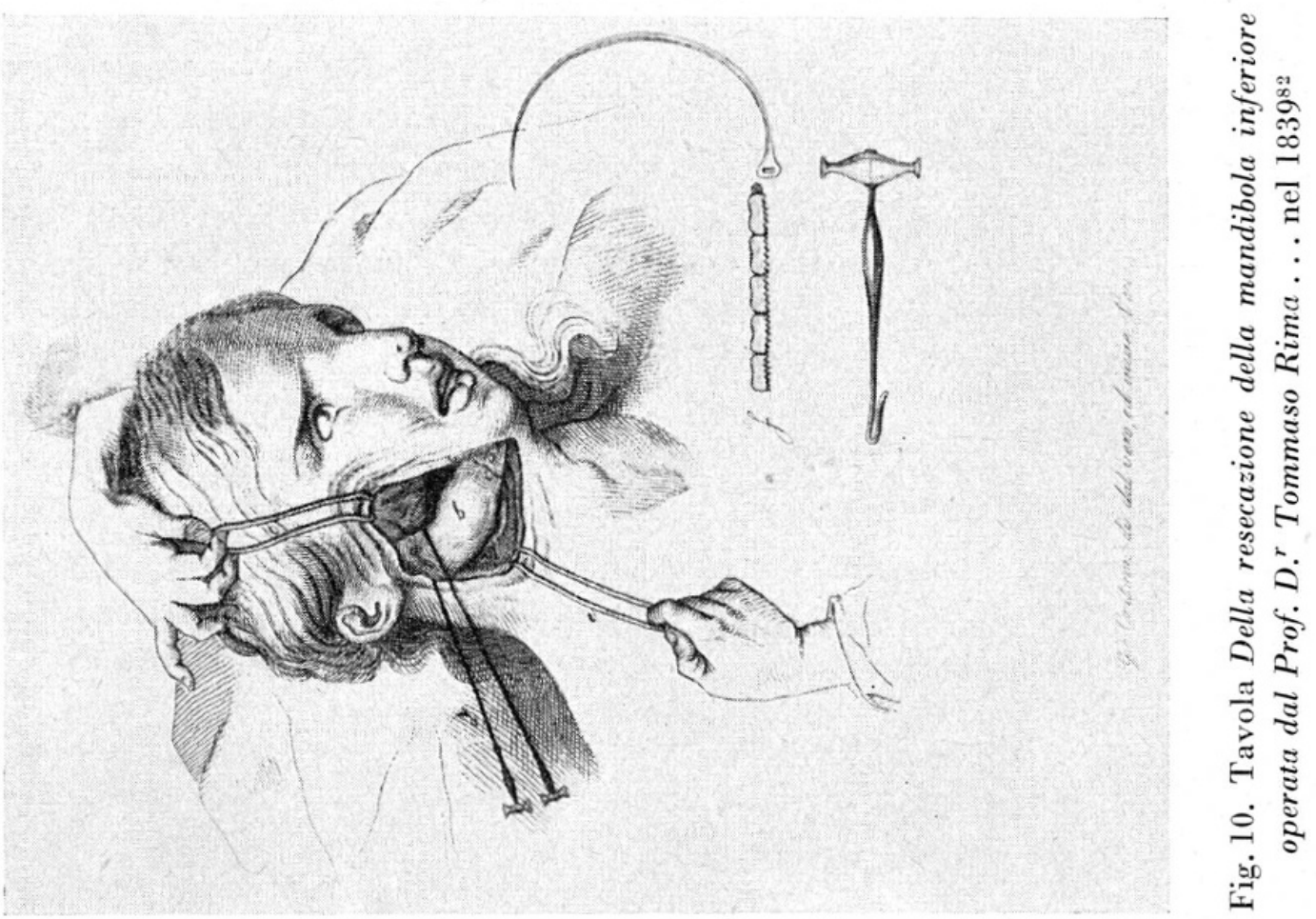


pagnata da quella somma prudenza, e amorevolezza propria del di lei carattere ....».]

Abbandonando l'impiego di quella antica residenza degli Esarchi, ei rinunciava al maggior stipendio; ma pure il faceva per l'utile indiretto della sua famiglia amando di rientrare nello stato; ed il faceva deciso, dopo un lungo pellegrinare, di stabilire invariabilmente in Venezia l'ultimo suo domicilio. A questo si trasportava nel giugno del 1822, e dal consiglier Governativo, Protomedico D. ${ }^{\mathrm{r}}$ Aglietti $^{60}$ veniva con speciale formalità messo in possesso della sua carica nell'ospitale il giorno 3 luglio. Egli si è fatto scrupolo di adempire con zelo ed esattezza a' suoi doveri, spesso anche in onta alla sua mal ferma salute. Se abbia corrisposto al comun voto, ed abbia ben meritato della città fia dovere il dirlo con imparzialità chi di lui scriverà dopo morte. Il defunto Professore Cesare Ruggieri ${ }^{61}$ lo dichiarava il solo chirurgo di Venezia ch'egli credesse tale; e volle che andasse ad assisterlo nell'ultima sua malattia, consultando coi Professori Bonato e Caldani ${ }^{62}$.

Poco dopo il suo arrivo veniva eletto membro ordinario dell'Ateneo di Venezia, e di Treviso; e vi faceva nel primo importanti letture, alcune delle quali pubblicate con le stampe. Non volle chieder autorizzazione d'accettare esteri diplomi che gli furono spediti.

Nel 1805-1808 fu stampata con note la sua traduzione ${ }^{63}$ dell'opera di Dufouart sulle ferite d'arma da fuoco ${ }^{64}$; opera di cui l'Italico ministero della guerra ne acquistava 250 copie per distribuirsi ai chirurghi d'armata.

$\mathrm{Fu}$ inventore di una macchina, quanto semplice, altrettanto utile per curare le fratture delle estremità inferiori ${ }^{65}$. Venne all'atto dell'invenzione addottata dal Cav. Paletta ${ }^{31}$ ed Assalini3 ${ }^{34}$ in Milano, dal Mina ${ }^{66}$ in Cremona, dal Piccinelli ${ }^{67}$ in Bergamo, dal Bolardi e Solera ${ }^{39}$ in Mantova, oltre che introdotta in tutti gli ospitali militari del cessato Regno d'Italia, compreso quello della R. Marina.

Nel fascicolo di maggio del 1819 dei commentarj di Padova leggesi una sua memoria sopra la cataratta ${ }^{68}$. Negli opuscoli scientifici di Bologna del 1821 esiste una sua memoria sul perfezionamento del processo operativo per l'estirpazione dei testicoli scirrosi ${ }^{54}$. Nel giornale del D. ${ }^{r}$ Namias del . . 1836 leggesi una memoria sul coléra che dominava in Venezia ${ }^{69}$.

Nello stesso giornale ${ }^{70}$ del 1837 trovansi registrate due memorie, lette nell'Ateneo di Venezia, una sul varicocele, l'altra sopra la cura radicale delle varici alle gambe. Rende nota in questa la sua opinione sul movimento inverso del sangue venoso, come causa, ed effetto delle varici, provandolo con dimostrazioni di fatto, e dando spiegazione del modo con cui, e per cui possi aver luogo la radicale guarigione. [Ne aveva già dato cenno a quel scientifico consesso nella lettura del 29 dicembre $1825^{71}$.]

Varj articoli pubblicava sotto la lettera iniziale $R$, e tre col suo nome nella Gazzetta Privileggiata di Venezia nel 1837, sul coléra, sulla idrofobia, sulla cura dell'ernia incarcerata ${ }^{72}$; e altri diversi sul giornale del Namias ${ }^{73}$. 


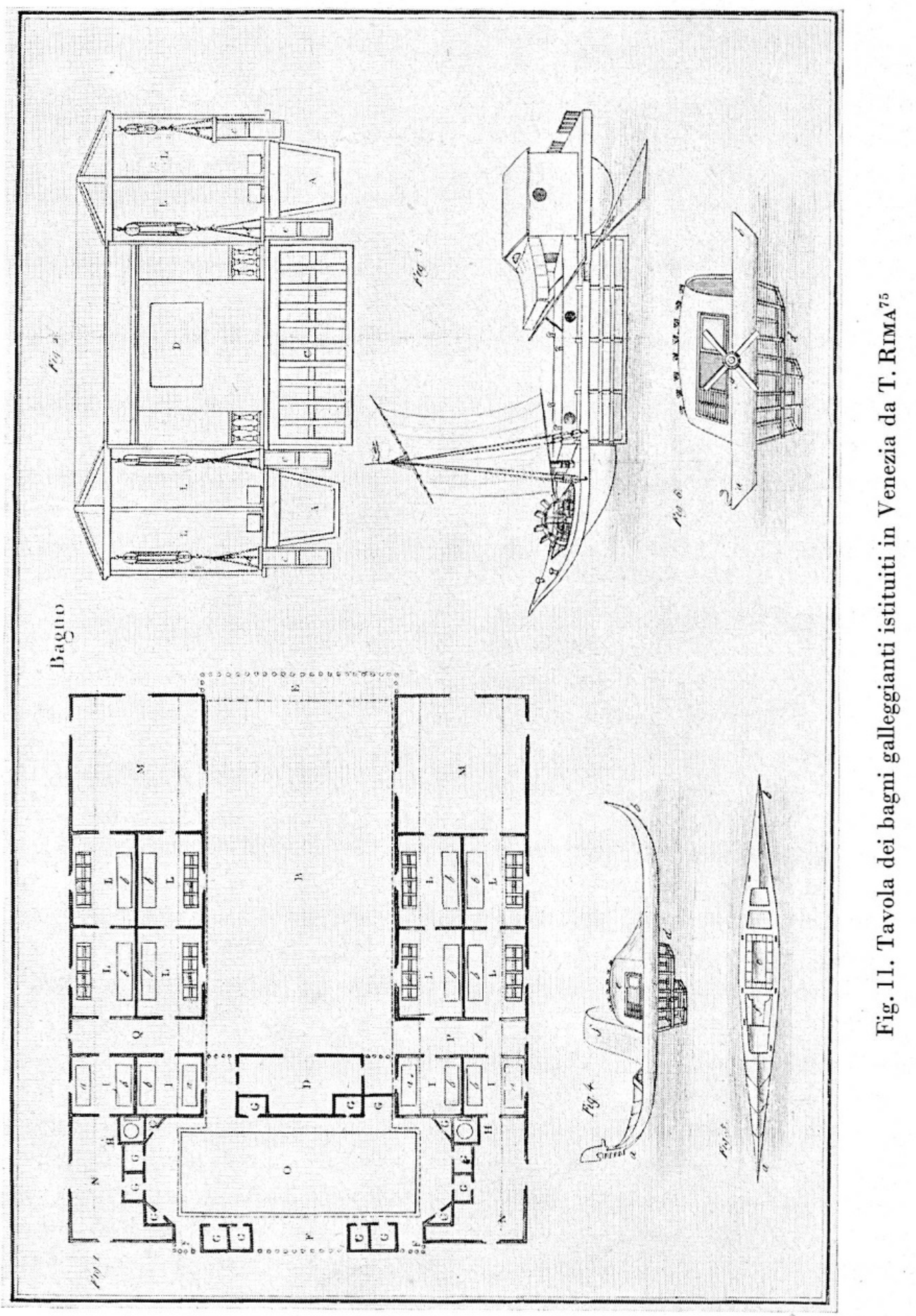


Con grave suo dispendio ergeva in Venezia un grande stabilimento di Bagni galleggianti $^{74}$, e ne otteneva da S.M. il privilegio esclusivo nel 1833. Ad esso univa un numero d'altri piccioli galleggianti coi quali si prende bagno anche girando in laguna come venne più circonstanziatamente pubblicato dall'Editore del Dizionario Tecnologico d'arti e mestieri, Venezia $1836^{75}$. E cotesta una istituzione che chiamerà sempre forestieri in Venezia; e tra gli abitanti stessi acquisterà pregio maggiore quanto più si conoscerà l'immensa diversità tra i vantaggi del bagno nell'acqua naturale e corrente, e il bagno in circoscritti recipienti. Per giudizio del R. Istituto ottenne da S. M. l'Imperatore Francesco I. ${ }^{\mathrm{mo}}$ la medaglia d'argento nel 1833: e nel pubblico concorso del 1835 il maggior premio, cioè la medaglia d'oro di S.M. Ferdinando Primo ${ }^{76}$.

Si prestava gratuitamente in una apposita stanza ai poveri, ed a chiunque nell'atrio dell'ospitale il chiedesse d'un suo consiglio, ed anche di una operazione; nè infrequente era il caso che provvedesse $\mathrm{i}$ bisognosi di mezzi pecuniarj per pro(v)vedere l'occorrente. Operava egli nei casi i più gravi, ma permetteva che sotto la sua direzione $i$ giovani alunni eseguissero quelle operazioni che repplicate volte s'erano sotto i suoi occhi addestrati a compiere sul cadavere. Era convinto per massima che questa era la via per renderli valenti operatori.

Indifferentemente di qualunque divisa fossero coperti i feriti, intrepido aveva sempre prestato i più urgenti soccorsi sul campo di battaglia non sempre scevro di pericoli anche per gli ufficiali di sanità nelle vicende della guerra. Ma sentiva un ribrezzo (dal dovere soltanto superabile) allorquando veniva chiamato alla assistenza di un pazzo. Aveva in ispreggio quelli (che) si piacevano di ritrarre spasso dal conversare con gl'infelici che avevano perso la ragione. Abborriva poi gli altri che per barbara curiosità accorrevano spettatori volontarj al supplizio d'un reo. Reduci da quello spettacolo cui non reggeva il chirurgo, chiamavano ${ }^{77}$ questo crudele nel vederlo ridare la vita ad un uomo!

Se molti venivano dall'esterno al di lui domicilio per esservi curati, ed operati, altri pur molti il chiamavano a consulto, e ad operare in lontani paesi. Egli vi accorreva, e per ritornare sollecitamente al dovere della propria residenza anche nel mal tempo cimæntava non senza qualche imprudenza i suoi giorni.

[Per parlarne di uno nel primo viaggio della diligenza veloce da Trieste a Venezia nella notte del 15 novembre, un'improvvisa alluvione per dirottissima pioggia trasformava in rapido torrente gran porzione di strada fra Palmanuova ed Udine. Sotto il villaggio di Santo Stefano veniva immersa la carrozza a segno da non poter progredire per l'acqua ognor crescente. Uno dei postiglioni si era salvato tagliando i tiranti del suo cavallo col titolo di andare a chieder soccorso. Intanto gli altri cavalli venivano a forza rivolti indietro, e doveva seguirli la carrozza che non aveva che mezza voltata. L'altro postiglione viene trascinato dalla corrente e perisce a mezzo miglio di distanza annegato. Fortunatamente la ca(r)rozza voltò senza esser rovesciata, e piegò nel fosso sino a che dopo due ore 
di crudele aspettare furono gettate delle tavole sul cielo della ca(r)rozza formando un ponte per sortire i passaggieri, il condut tore e l'impiegato(?) chel'accompagnava.]

Medico ed amico di S.E. il Generale Conte Caffarelli ${ }^{78}$ Ministro della guerra, fece del bene a molti: ma in pochi trovò corrispondenza e gratitudine. Fu molto gajo nella sua prima gioventù; ma l'importanza del servizio che ha dovuto sostenere specialmente nei primi tempi nell'armata esaltata da' principj reppubblicani, lo resero abitualmente serio e severo. Conservò sempre come accade ne' Svizzeri l'amore del paese nativo; ma le vicende della sua famiglia gli allontanarono sempre il pensiero di farvi stabile ritorno.

Diceva che ogni ammalato era in diritto di conoscere il proprio stato. Se generalmente reputavasi inurbanità il farglielo conoscere quando il prognostico non poteva esser felice; era secondo lui riprovevole tradimento il lasciarglielo ignorare. Disapprovava questo contegno de' suoi colleghi verso di lui stesso che dal 2 marzo 1838 presumeva prepararglisi un vizio nell'arco dell'aorta e lungo la carotide sinistra. Forse nasceva da una sproporzione di circolazione tra il sistema arterioso, ed il venoso. I suoi amici lo giudicheranno dopo la di lui morte. Gli è indifferente che lo verifichino o no con la sezione del cadavere ${ }^{79}$. Unito alla decadenza dell'età, risentì gli effetti d'una caduta sulle scale dell'ospitale nel giorno in cui S.E. il nuovo Governatore fece la sua prima visita all'ospitale il . . giugno 1841. Gli parve sentire i premordi d'una paraplegia che dicesi traumatica.

Non si è mai pentito d'aver tacciuto. L'aver parlato ha contrariato affatto l'ordine delle cose nella sua carriera.

Considerato sotto i rapporti famigliari, egli conservò sempre grata riconoscenza all'ottimo suo Zio Remigio Rima, onestissimo Negoziante in Lugano, che prese amorosa cura di lui nei disastri della famiglia. Ad esso, ed a sua moglie Maria Antonia, saldate alcune passività, pagava spontaneo un vitalizio in contrassegno di grata riconoscenza. Ad altri suoi Zii ch'ebbero neppure l'apparenza di benefattori, e che dichiarati creditori perdenti nell'atto ch'entravano al possesso d'ogni suo patrimonio, egli pagava nel 1810 e capitale e frutti ed interessi di questo. Rimborsava di più col proprio di quanto avevano pagato per il di lui maggior fratello, senza che questi ne avesse goduto per la loro indecisione il competente profitto. Alla figlia del defunto suo fratello faceva dare educazione in un chiostro dell'Umbria e raccoglieva qual figlia in sua casa, dopo la soppressione dei monasterj, e riteneva sino a che vedovo egli stesso nell'incertezza delle vicende della guerra nel 1813 era astretto a rimandarla alla madre in Roma; come era obbligato ad affidare ad estere mani le sue bambine.

A queste procurò una madre amorosa nel 1814 nella Marchesa Teresa Lalatta di Parma, vedova del conte Giov. Battista Mozzi ${ }^{48}$ di Bergamo, ottenendola per sua moglie. Una delle sue figlie ${ }^{80}$ ebbe un ottimo compagno nel D. ${ }^{r}$ Paolo Pinelli figlio del vivente D. ${ }^{r}$ Orazio, famiglia che suol dirsi degli Asclepiadi di Zara per la serie dei medici che si successero sempre; divenuto ora da poco Medico Diret- 
tore di quel civico ospitale. L'altra ${ }^{81}$ a lui superstite è tutt'ora nubile. Per questa solo avrebbe bramato qualche anno di più di vita, purché meno disgraziata. Ma non sente rimorsi se il matrimonio non si verificò in chi ne fece la domanda. Prevedeva nel suo amor paterno che non sarebbe stata felice; la felicità di sua figlia era il solo scopo a cui bramava giungere.

Se nel destino era scritto ch'egli fosse rimasto un'altra volta vedovo; appena provvista la figlia, era suo divisamento di terminare i suoi giorni Religioso di San Giovanni di Dio tra i Fatebenefratelli se l'istituto permetteva che fosse stato come tale accolto sebbene nell'età già avvanzata.

Nel marzo 1839 operò felicemente la resezione della mandibola con utili modificazioni, come alla memoria del Dr. Marconi ${ }^{82}$. Fece pure felicemente in Mestre l'operazione cesarea, salvando madre, e figlio ${ }^{83}$.

Ignoro se l'invenzione d'un suo strumento per l'estrazione del calcolo vescicale col taglio ipogastrico otterrà l'approvazione del R. Istituto ${ }^{84}$.

Col giorno 23 agosto 1841 a pieni voti fu eletto dall'Ateneo di Venezia Membro Onorario dell'Ateneo stesso. Si è grato a quel consesso perché di tutti gl'intervenienti in quella seduta la sua elezione non fu contrariata da un sol voto. Tutti hanno votato favorevolmente.

\section{Divergenze (?) parziali}

Da sistemarsi se è possibile in giorni di calma.

${ }^{1}$ Considérations sur la cause prochaine des varices des membres inférieurs et sur leur traitement radical; mémoire lu à l'Athénée médical de Venise; par M. Rima, chirurgien du grand hôpital de cette ville, Gazette Médicale de Paris 5, 427 (1837). Cf. la nota 70.

${ }^{2}$ p. 127-31 del vol. II (1842) delle Annotazioni anatomico-patologiche e pratiche intorno le chirurgiche malattie, Venezia 1842-1845, in 4 vol.

${ }^{3}$ Elémens de pathologie chirurgicale, Paris 1844, t. I, p. 256: «M. RiмA, chirurgien au grand hôpital de Venise, trouve la cause prochaine des varices des membres abdominaux en dehors de toutes les causes déjà indiquées. Cette cause prochaine, suivant lui, consisterait dans un mouvement rétrograde du sang veineux. Voici ses raisons: $1^{\circ} \mathrm{Si}$ l'on excise sur le vivant une portion d'une veine variqueuse, on voit le sang jaillir du bout supérieur, comme d'une artère. $2^{\circ}$ Chez les personnes dont les varices ont été occasionnellement produites par une jarretière trop serrée au-dessous du genou, on voit les veines se dilater plus au-dessus qu'au-dessous de l'étranglement. $3^{\circ}$ Lorsqu'on pratique soit la ligature, soit l'excision d'une veine variqueuse, on voit les groupes variqueux placés au-dessous s'affaisser, se ramollir et disparaître, tandis que les varices de la même veine placées au-dessus restent stationnaires ou bien augmentent, ce qui ne devrait pas avoir lieu si le sang du même vaisseau marchait de bas en haut, comme dans l'état normal. 4 L'anatomie pathologique viendrait encore, dit-il, confirmer sa manière de voir.»

${ }^{4}$ Über die Unterbindung der Vena saphena magna bei Unterschenkelvaricen, Bruns' Beiträge zur klinischen Chirurgie 7, 194-210 (1891). 
${ }^{5}$ Nel centenario della dottrina di Tommaso Rima su le varici, mentre si scopre a lui un ricordo marmoreo e si inaugura il III ${ }^{\circ}$ Congresso della Società Italiana di Storia delle Scienze Mediche e Naturali. Orazione tenuta nell'aula magna dello Spedale civile di Venezia dal Sen. Dr. Davide Giordano, chirurgo primario dello spedale e presidente della Società, XIII settembre MCMXXV, Venezia 1925, in-4, di p. 48 e tav. 1. L'orazione è riprodotta a p. 270-95 di D. Grondano, Scritti e discorsi pertinenti alla storia della medicina e ad argomenti diversi, Milano 1930, di p. 630. Cf. pure D. Giondano, L'Italia nella chirurgia delle varici, Rassegna Clinico-Scientifica (Milano) 14, 343-8 (1936). Per la storia dell'Ospedale civile di Venezia, che è tuttora situato, come all'epoca del Rima, nella Scuola Grande di S. Marco, capolavoro del Rinascimento veneziano, cf. D. Giondano, L'Ospedale Civile di Venezia, Scritti e discorsi .... p. 94-105; L. ZaNaLd, Notizie preliminari per una storia documentata dell'Ospedale Civile di Venezia, Venezia 1950, di p. 53 e fig. 18.

${ }^{6}$ A. Minich, Commemorazione del prof. Michelangelo Asson letta all'apertura della scuola pratica di medicina e chirurgia, Giornale Veneto di Scienze Mediche 29, 609-56 (1878).

${ }^{7}$ Elogio del Professore Tommaso Rima pronunciato dal Dottor Michelangelo Asson nell'Ateneo di Venezia, Giornale per servire ai progressi della Patologia e della Terapeutica 3, 310-27 (1843). Il Rima morì il 26 febbraio 1843 e il giorno successivo M. Asson fece apparire sulla Gazzetta Privilegiata di Venezia una breve necrologia del Maestro. Altro breve Cenno necrologico su T. Rima a cura di Alessandro Tassinari apparve su Il Gondoliere del 15 marzo 1843.

${ }^{8}$ In morte del Prof. Dott. Tommaso Rima chirurgo primario anziano nell'Ospitale Civile Provinciale di Venezia, ec.; discorso del Dott. Luigi Nardo ..., Memoriale della Medicina contemporanea 9, 273-84 (1843). Una nota avverte che «Il discorso fu letto nel dì 27 marzo, trigesimo della morte, nella Chiesa interna dell'Ospitale durante l'uffizio funebre». L'estratto (Venezia 1843, di p. 16) porta un titolo lievemente variato Tributo alla memoria del Prof. Dott. Tommaso Rima chirurgo primario anziano nell'Ospitale Civile Provinciale di Venezia, del Dottor Luigi Nardo..., mentre il testo è preceduto dalla dedica «a/Paolo zannini/Medico e letterato/ Primario anziano Nel civico ospitale / A GUi MERito / QUANDo L'Istituto diresse / VeNEzia OTTENEVA / L'Illustre MAESTRO OPERATORE / TOMMASO RIMA ..."

9 Nato dal matrimonio di Paolo Pinelli con Elisa Rima, figlia di Tommaso. Un breve necrologio di Orazio Pinelli è contenuto in D. Giondano, Scritti e discorsi pertinenti alla storia della medicina e ad argomenti diversi, Milano 1930, p. 507-8.

${ }^{10}$ Ricerche, scoperte e invenzioni di medici svizzeri. Esposizione di libri e manoscritti organizzata con l'appoggio della Ciba Société Anonyme, Basilea, dalla Biblioteca Cantonale, Lugano, Basilea 1947, di 137 p. +8 p. di Appendice.

11 A. ZANoLI, Sulla milizia cisalpino-italica: cenni storico-statistici dal 1796 al 1814, Milano 1845, vol. 2 e album in f. ${ }^{\circ}$ di tav. 5. - F. Turotti, Storia dell'armi italiane dal 1796 al 1814, Milano 1855-1858, vol. 3.

12 Nel manoscritto è aggiunto d'altra mano «morto il 26 febbraio $1843 »$.

${ }^{13}$ Continuazione e compimento del dizionario storico-ragionato degli uomini illustri del Canton Ticino, Lugano 1811, p. 61-4.

${ }^{14}$ Dizionario storico-ragionato degli uomini illustri del Canton Ticino, Lugano 1807, p. 153-3.

${ }^{15}$ S. Spirito in Sassia, Ss. Salvatore in S. Giovanni al Laterano, S. Giacomo in Augusta (o degli Incurabili), S. Maria della Consolazione. Per la storia degli ospedali romani, 
ef. A. Canezza e M. Casalini, Il Pio Istituto di S. Spirito e Ospedali Riuniti di Roma, Roma 1933.

${ }^{16}$ Sul Celoni cf. S. De Renzi, Storia della Medicina in Italia, Napoli 1848, 5, 551-2; G. A. Oldelli, Continuaz. e compim. del Dizion. stor. ..., nota 2 a p. 62. Il Prof. Pietro de ANgelis (Direttore della Biblioteca Lancisiana, Roma) mi ha poi cortesemente comunicato che nell'atrio della Direzione dell'Ospedale di S. Giovanni in Laterano si trova la seguente lapide non datata: CELONI tommaso MARIA / CHIRURgo PRIMARIo / LETTORE DI ANATOMIA E CHIRURGIA / IN QUESTO OSPEDALE / PUBBLICò VARIE OPERE / DI CHIRURGIA E MEDICINA FORENSE/E FU RIPUTATO DAL MORGAGNI/UNO DEI PIÙ VALENTI CHIRURGHI / DEL SUO TEMPO.

${ }^{17}$ Giuseppe Sisco (1748-1830), nato a Bastia, fu chirurgo primario negli ospedali di Roma e occupò la cattedra di medicina operatoria e poi di clinica chirurgica alla Sapienza.

${ }^{18}$ Giongio Bonelli (1724-1803), nato a Vico presso Mondovì, fu lettore pubblico di medicina pratica alla Sapienza e ricoprì più volte il protomedicato.

${ }^{19}$ Domenico Pino (1767-1828), cui il Rima dedicherà la traduzione italiana del trattato di P. Dufouart (cf. nota 63).

${ }^{20}$ In data 18 settembre 1802 «Rezia Giacomo è creato direttore di sanità militare. Dell'U e Solenghi, che erano del consiglio di sanità, sono dipendenti da lui, il primo come medico, ed il secondo come chirurgo superiore dell'esercito" (ZANoLI, vol. I, p. 10-1). Il Dell'U è verosimilmente lo stesso citato a p. 129 da P. CAPPARONI, Lazzaro Spallanzani, Torino 1941.

${ }^{21}$ Giacomo Rezia (1745-1825), dal 1772 professore d'anatomia e chirurgia a Pavia e dal 1783 al 1796 professore di fisiologia e patologia generale nella stessa Università.

${ }^{22}$ Frase non terminata.

${ }^{23} \mathrm{Su}$ questa epidemia cf. A. Corradi, Annali delle epidemie occorse in Italia dalle prime memorie fino al 1850, Bologna, 4, 550-60 e 563-8 (1876); 7, 949-52 (1892).

${ }^{24}$ Cf. Turotti, loc. cit., vol. 1, p. 556.

${ }^{25}$ «Vienna» aggiunto in un secondo tempo; ma si tratta verosimilmente della pace di Presburgo (26 dicembre 1805).

${ }^{26}$ Carlo Cocchettr (1763-1834), convinto antirasoriano, medico in capo dell'Ospedale militare di Milano, che allora occupava i locali dell'ex-monastero cisterciense di S. Ambroxio, celebre pei due armonici chiostri bramanteschi: dal 1931 è sede dell'Università Cattolica del Sacro Cuore.

${ }^{27}$ Carlo Cairoli (1776-1849), padre degli eroici fratelli, insegnò a Pavia ostetricia (18091819) e istituzioni chirurgiche (1810-1834). Pel suo intervento sulle varici cf. lo scritto del VoLPI citato nella nota 38.

${ }^{28}$ Everard Hoмe (1763?-1832), Practical observations on the treatment of ulcers on the legs, considered as a branch of military surgery; to which are added some observations on varicose veins and piles. Second edition, considerably enlarged, London 1801, di p. 362 ( ${ }^{\text {a }}$ ediz. 1797).

${ }^{29}$ La lacuna lasciata dal Rima fu già colmata da G. A. Oldelli (p. 63 della Continuaz. e compim. ${ }^{13} \ldots$ ), il quale precisò la data: 11 giugno 1807. In questo senso parlano anche $\mathbf{i}$ documenti sulla concessione della cittadinanza italiana al Rima conservati all'Archivio di Stato di Milano (Atti di Governo, Albinaggio p. m., cart. 90, fasc. 11). 
${ }^{30}$ Frase non terminata. Il dispaccio è però datato 2 luglio 1807 , come risulta dalla minuta conservata all'Archivio di Stato di Milano (loc. cit. nella nota 29).

${ }^{31}$ Giovanni Battista Pa(L)Letta (1748-1832), chirurgo-capo e professore d'anatomia all'Ospedale Maggiore di Milano. Per la sua dottrina sulle varici cf. Sopra le varici, considerazioni del Cav. Giambattista Paletta, professore di anatomia e chirurgia, membro onorario, Esercitazioni scientifiche e letterarie dell'Ateneo di Venezia 1, 89-97 (1827).

32 Giovanni Battista Monteggia (1762-1815), allievo del Palletta, celebre chirurgo e professore di chirurgia all'Ospedale Maggiore e alla Maternità milanese. Per la sua dottrina sulle varici ef. S. Piccini, G. B. Monteggia e la chirurgia delle varici, Milano 1940, di p. 26.

${ }^{33}$ Giovanni Rasoni (1766-1837), il celebre maestro della dottrina del «controstimolo», fu nominato professore di clinica medica presso l'Ospedale Maggiore (1806) e presso l'Ospedale militare di S. Ambrogio (1807) in Milano. Nella notte dal 3 al 4 dicembre 1814 fu arrestato dagli Austriaci per aver partecipato alla «cospirazione militare» e rimase in carcere, prima a Mantova e poi a Milano, fino al 9 marzo 1818. Al momento dell'arresto versava in estrema povertà e durante la lunga prigionia lasciò in gravissime ristrettezze finanziarie la giovane figlia Sabina. Cf. A. Monti, Giovanni Rasori nella storia della scienza e dell'idea nazionale, Corsi autunnali per Italiani e Stranieri tenuti nella R. Università di Pavia: lezioni e conferenze dell'anno 1928. Pavia 1929, p. 5-143. - Sulla dottrina rasoriana del «controstimolo» e più generalmente sulle correnti dottrinarie della medicina italiana all'epoca del Rima, cf. l'importante studio storico-critico di A. CAzzANIGA, La grande crisi della medicina italiana nel primo Ottocento, Milano 1951, di p. 122; pubblicato anche a puntate nei volumi 4, 5, 6 (1948-1950) della rivista Castalia.

${ }^{34}$ Paolo Assalini (1759-1840), fu nominato nel 1807 professore di clinica chirurgica presso l'Ospedale militare di S. Ambrogio in Milano. Cf. A. F. LA Cava, Assalini, Trieste 1947, di p. 98.

${ }^{35}$ Seguivano le parole «che lo diresse al collega in Mantova» poi cancellate.

${ }^{36}$ Seguivano le parole «secondo i principi di Home» poi cancellate.

${ }^{37}$ Tommaso Volpi (1761-1822), chirurgo primario dell'Ospedale di Pavia, supplì nel 1804 lo Scarpa nell'insegnamento della clinica chirurgica, di cui fu nominato professore ordinario nel 1817.

${ }^{38}$ Lo scritto del VolpI, Della cura radicativa delle varici alle gambe: cenni pratici, si trova a p. CXXXII-CLXX del supplemento al vol. VIII (1811) degli Elementi di chirurgia di Augusto Gottlieb Richter ... Recati sulla seconda edizione dall'Idioma Tedesco nell' Italiano ed arricchiti di varie annotazioni da Tommaso Volpi ..., Pavia, 1794-1811, in 8 vol. Lo stesso scritto del Volp fu riprodotto nel Saggio di osservazioni e di esperienze medicochirurgiche fatte nello Spedale Civico di Pavia da Tommaso Volpi..., Milano, 1814, vol. II, p. 133-82.

39 Giuseppe Solera.

${ }^{40}$ Sulla epidemia oftalmica di Ancona ef. L. Belloni, Una ricerca del contagio vivo agli albori dell'Ottocento, Gesnerus 8, 15-31 (1951).

${ }^{41}$ Anche qui la memoria delle date fa difetto al Rima, dato che la Storia del Vasani apparve nel 1816.

${ }^{42}$ Francesco Vasani (1782-1850), medico veronese, autore della Storia dell'ottalmia contagiosa dello Spedale militare d'Ancona: origine e natura di quel contagio ed alcuni cenni 
intorno ai comuni errori nella terapeutica dell'ottalmia cronica (Verona 1816, di p. 140) e della Risposta di F. Vasani a ciò che lo riguarda nei Cenni del Dr. Omodei sull'ottalmia contagiosa d'Egitto e sulla sua propagazione in Italia (Verona 1818, di p. 47).

${ }^{43}$ Paolo Bongrovanni (1777-1827) percorse la carriera nell'esercito napoleonico e fu per cinque anni chirurgo in capo e professore di clinica chirurgica nell'ospedale militare d'Ancona. Nel 1819 ottenne per concorso la cattedra d'ostetricia all'Università di Pavia.

${ }^{44}$ C. G. Annibale Omodei (1779-1840), che aveva ricoperto la carica di medico consulente al Ministero della Guerra del Regno Italico, scrisse in risposta al Vasani i Cenni sulla ottalmia contagiosa d'Egitto e sulla sua propagazione in Italia, Annali di Medicina straniera 4 (continuaz.), 104-44, 222-88, 324-74 (1816). L'opera figura anche in fascicolo a parte (Milano 1816, di p. 159) e fu tradotta in tedesco (Frankfurt am Main 1820).

${ }^{45}$ Pubblicò invece integralmente (Biblioteca Italiana 7, 97-101 [1817]) due lettere di A. Scarpa, la prima indirizzata al Ministro della Guerra il 13 novembre 1812 e mandata in copia al Rrma quale ispettore di sanità in Ancona, la seconda diretta al Rima stesso il 14 dicembre 1812.

${ }^{46}$ Gioacchino Murat (1767-1815), re di Napoli dal 1808, che doveva scontare la sua illusione fucilato al Pizzo di Calabria il 13 ottobre 1815.

${ }^{47}$ Giuseppe Prina (1766-1814), grazie alla sua carica, fu ritenuto responsabile della pressione fiscale durante il periodo napoleonico e, alla caduta del Regno Italico, fu ucciso dal furor popolare.

${ }^{48}$ Riporto un brano del testo primitivo poi cancellato «... per assistere da vicino il conte Mozzi divenuto suo figliastro ... . Questo cognome ricorre anche verso la fine dell'opera.

${ }^{49}$ Il vicentino Bartolomeo Panizza (1785-1867), allievo di A. Scarpa, fu professore d'Anatomia all'Università di Pavia dal 1815 al 1864.

${ }^{50}$ Trattasi verosimilmente del riminese Nicola Morigr (1746-1836), laureato a Parma (1769), chirurgo all'ospedale di Piacenza (1780-1814), professore di Clinica chirurgica e d'Operazioni chirurgiche all'Università di Pavia (dall'agosto 1814 all'ottobre 1817), chirurgo-capo all'Ospedale Maggiore e professore d'ostetricia e chirurgo primario nell'Ospizio di S. Caterina alla Ruota in Milano (1817-1820), infine medico di Maria Luisa e insegnante di Clinica chirurgica all'Università di Parma. Nella Risposta... sull'operazione della pietra... (Ravenna 1820) il RIMA, difendendo il proprio metodo operatorio, lo dice usato anche da «uno dei più fortunati operatori il prof. MoRIGgi (sic!) già clinico di Pavia, ed ora Ispettore generale degli ospitali in Parma» (p. 21). Inoltre M. Asson (Elogio ..., p. 319) parafrasando questo brano della Necrologia del Rima trascrive «Morigi».

${ }^{51}$ Antonio Scarpa (1752-1832), professore d'anatomia e di clinica chirurgica e dal 1812 direttore della Facoltà medica di Pavia.

52 Siro Borda (1761-1824), fervente seguace delle dottrine rasoriane, fu professore di materia medica all'Università di Pavia.

${ }_{53}$ Trattasi probabilmente di Pietro Tamburini (1737-1827), da Brescia, sacerdote, capo del giansenismo italiano, che il 28 novembre 1778 fu chiamato dal conte di Firmian alla cattedra di Teologia morale dell'Università di Pavia.Nello stesso Ateneo ricoprì in seguito, con alcune interruzioni dovute in parte alle vicende politiche del periodo napoleonico, altre cattedre delle facoltà di teologia, di filosofia e lettere e di giurisprudenza. 
53a A tali concorsi, e precisamente a quelli per le cattedre d'oculistica e d'ostetricia vacanti in ambedue le Università del Regno Lombardo-Veneto (Padova e Pavia), si riferiscono tre autografi di Bartolomeo Panizza ${ }^{49}$ conservati all'Archivio di Stato di Milano (Raccolta degli autografi, Medici, cart. 217, fasc. 28). Nel primo documento, non datato e intitolato Giudizio del Prof.e Panizza sopra gli scritti dei tre concorrenti alla Cattedra delle malattie degli occhj, l'illustre anatomico, vagliate le risposte dei candidati Pugliati, Flarer, Donegana ai tre problemi proposti (ottalmia scrofolosa, ipopion, metodi operativi della cataratta), giunge alla conclusione che nessuno di essi «ha le qualità necessarie per occupare la Cattedra di cui si tratta». Ben diversamente suona, nel secondo documento, il giudizio espresso dal Panizza sul Rima: «St. ${ }^{0}$ Sig. ${ }^{r}$ Direttore/Dietro l'invitazione di V.S.I. io esaminai gli scritti del Sig. ${ }^{r}$ Dott. ${ }^{\mathrm{r}}$ RIMA, concorrente alla Cattedra d'Oculistica, vacante in ambedue le Università del R. Lombardo-Veneto, e mi fo sollecito a parteciparle, che al mio rapporto trasmessole sugli altri tre aspiranti, Flarer, Pugliati, e Donegana, unisco il seguente giudizio sul soggetto in questione. Il Sig. ${ }^{r}$ D. ${ }^{r}$ Rima nella soluzione dei tre quesiti, usò sempre uno stile semplice, e puro, con molto ordine, e chiarezza d'idee, caratteri invero essenziali per un aspirante alla pubblica Istruzione. Ho poi la compiacenza d'assicurare, che più d'ogni altro à soddisfatto a quanto ricercavano le dimande, benchè non ignori aver egli fatta qualche ommissione, come p. e. trattando dell'ottalmia acuta scrofolosa poco ragionò sui sintomi che l'accompagnano, e sulle sue cause occasionali; così pure nel $2 .^{\text {do }}$ quesito poco parlò degli esiti dell'ipopion. Tutte queste lacune vennero appieno ricompensate colla soluzione del terzo quesito, ove ad una peregrina, e sensata erudizione egli aggiunse una analisi ragionata sopra i varj metodi, colla quale fece conoscere quanto egli sia versato nella Teoria, e pratica delle operazioni. In sequela di tutto ciò, e dei di lui meriti per varie produzioni letterarie, non che per gli incarichi pubblici, che con tanto decoro, ed onore egli coprì, non esito punto a giudicarlo idoneo ad occupare onorificamente la suddetta Cattedra. 28 Giugno 1818 Pavia/Bartolomeo Panizza Pr.e/d'Anatomia Umana.»

Nel terzo autografo, che è di poco posteriore (Addì 18 luglio 1818, Pavia) e s'intitola Giudizio del Prof.e Panizza sugli scritti dei sette concorrenti alla Cattedra d'Ostetricia, l'illustre anatomico, vagliate le risposte dei candidati (Fenini, Pugliati, Bongrovanni ${ }^{43}$, Molina, Billi, Rima, Moro) ai tre problemi proposti (il primo riferentesi al parto, e gli altri due all'obliquità dell'utero gravido e all'operazione cesarea), così conclude: «Riassumendo quanto esposero $\mathbf{i}$ candidati nella soluzione dei tre quesiti, e paragonando il merito d'ognuno con quello degli altri, avuto in riflesso l'esame da loro sostenuto a voce, io mi trovo in dovere, di nominare per il p. ${ }^{\circ}$ il Sig. ${ }^{r}$ Molina, indi Rima, il terzo BiLLI.» In particolare, il Rima ottenne il seguente giudizio: "Il Sig. ${ }^{r}$ Dott. ${ }^{\mathrm{r}}$ Rima nulla lascia a desiderare sulle prime parti del $1^{\circ}$ quesito, mentre sulle consequenze relativamente alla madre, ed al bambino, nominò evvero tutto ciò, che in genere si può dire rapporto le medesime, ma non si estese a ragionare su quelle, che accadere possono, e all'aborto, ed al parto maturo, prematuro, e doppio. Esposte tutte le opinioni che vi furono circa la causa prossima delle obliquità dell'utero, egli passa a descrivere li segni delle singole obliquità. Tra i sintomi delle obliquità laterali non nomina il torpore dell'arto corrispondente, il dolore ottuso o lancinante lungo il psoas, la regione inguinale, e la parte interna della coscia, l'edemazia o la varicosità dei vasi dell'arto suddetto; ed altri disturbi, che non di rado si palesano. Il Sig. ${ }^{r}$ concorrente, benchè sia stato un poco 
disturbato nella salute al momento della soluzione dell'ultimo quesito, pure laconicamente à soddisfatto a quanto esigeva il medesimo facendo saggiamente riflettere, che innanzi di passare all'operazione Cesarea in donna morta gravida è necessario d'osservare se il parto è fatibile per le vie naturali.»

${ }^{54}$ Sul perfezionamento del processo operativo per l'estirpazione dei testicoli scirrosi, Opuscoli scientifici (Bologna) 4, 233-43 (1820). Riprodotto a p. 839-44 del t. 40 (1847) del Dizionario classico di medicina, di chirurgia e d'igiene pubblica e privata composto da Adelon ... prima traduzione italiana di M. G. Levi ... con numerose giunte ..., Venezia (G. Antonelli). La traduzione francese Nouveau procédé opératoire pour l'extirpation des testicules squirreux con note e appendice a cura di Charles-Caspard Peschier (17821851) fu pubblicata in Mélanges de Chirurgie étrangère par une Société de Chirurgiens de Genève composée de MM. J. P. Maunoir, C. T. Maunoir. ... (Squirre, Cancer, Fongus, Tumeurs), Genève 1825, p. 281-331.

${ }^{55}$ Risposta del Professore Tommaso Rima all'anonimo autore della lettera critico-chirurgica sull'operazione della pietra da esso eseguita nel pubblico ospitale di Ravenna, Ravenna 1820, di p. 33.

${ }^{56}$ Il cadorino Marcantonio De MARChi (1760-1821) era stato nel 1812 nominato chirurgo in capo dell'Ospedale di Venezia.

${ }^{57}$ Antonio Codronchi, arcivescovo di Ravenna dal 1785 al $1826(\dagger)$.

58 Antonio Lamberto Rusconi (1743-1825), nominato nel 1816 cardinale e vescovo di Imola e nel 1820 legato di Ravenna.

${ }^{59}$ Un'altra lettera di servizio del Gonfaloniere, Conte DeLla Torre, al Rina, in data 3 luglio 1822 è riprodotta a p. 231 di G.E. Curatulo, L'arte di Juno Lucina in Roma; storia dell'ostetricia dall'origine fino al secolo XX, con documenti inediti, Roma 1901.

${ }^{60}$ Francesco Aglietti (1757-1836) da Brescia.

${ }^{61}$ Il cremasco Cesare Ruggieri (1768-1828), professore di chirurgia a Padova.

${ }^{62}$ Floriano Caldani (1772-1836), nipote del più celebre Leopoldo Marcantonio CalDANI (1725-1813).

${ }^{63}$ Analisi delle ferite d'armi da fuoco, e della loro cura di Pietro Dufouart, Ufficiale superiore di Sanità, e Chirurgo in capo dell'Ospital militare di Parigi. Traduzione con aggiunta di note di Tommaso G. Rima, Ufficiale di sanità di prima classe nell'Armata Italiana, Chirurgo Primario dell'Ospedal militare centrale alla destra del Pò, e della scuola militare del Genio, e dell'Artiglieria in Modena, Milano 1805-1808, 2 vol. di p. 301 e 219. Oltre alle note, il traduttore aggiunse l'articolo «Delle ferite d'armi da fuoco al collo» (vol. II, p. 136-44).

${ }^{64}$ Pierre Dufouart (1737-1813), Analyse des blessures d'armes à feu et de leur traitement, par Pierre Dufouart, Officier de santé supérieur et chirurgien en chef à l'hôpital militaire de Paris, Paris, An X. Vendémiaire, 1801, di p. 425.

${ }^{65} \mathrm{La}$ «correzione del metodo di curare le fratture oblique delle estremità inferiori con l'estensione permanente» e la sua adozione da parte di Palletta, Assalini e Piçcineldi furono già menzionate nel 1811 da G. A. Oldelli (Continuaz. e compim. del Dizion. stor. ..., p. 64). Anche M. Asson (Elogio ..., p. 323) parla di «un suo stromento per eseguire l'estensione permanente nelle fratture del femore» e L. Nardo (Tributo alla memoria ..., p. 14) precisa: «Nel 1808 inventava in Milano una macchinetta ad estensione permanente per la cura della frattura delle estremità inferiori, la quale veniva 
adottata dal Paletta, dall'Assalini, dal Piscianelli (sic!), dal Mina, dal Solera, e più diffusamente introdotta in tutti gli Ospitali militari d'Italia. Essa formò il soggetto di due Memorie lette all'Ateneo di Venezia.»

${ }^{66}$ Isidoro Camillo Mina (n. 1750), chirurgo e in seguito professore all'Ospedale Maggiore di Cremona. Cf. F. Rовоцотті, Storia e statistica economico-medica dell'Ospitale Maggiore di Cremona, Cremona 1851, p. 207-8.

${ }^{67}$ Giannantonio Piccinelut (1754-1831), chirurgo primario e ispettore all'Ospedale Maggiore di Bergamo.

${ }^{68}$ Riflessioni del dott. T. Rima Chirurgo principale d'armata e provvisoriamente I.R. CapoMedico-Chirurgo della Guarnigione di Legnago, sull'ago da cateratta, e sul metodo di cura da usarsi dopo l'operazione stessa proposti dal sig. dott. G. Schiantarelli chirurgo dell'ospitale delle donne in Brescia, Nuovi Commentarj di Medicina e di Chirurgia 3, 433-51 (1819). Il lavoro di Giuseppe Schiantarelli era apparso nella stessa rivista, stesso anno e volume, p. 3-26 e tav. 1. Le Riflessioni del Rima sono riprodotte a p. 44-54 del t. 6 (1843) del Dizionario classico di medicina ... di M.G. Levi ...

${ }^{69}$ Sul Coléra, cenni critici, Giornale per servire ai progressi della Patologia e della Materia medica (Venezia) 3, 27-46 (1835). In questo lavoro, il Rima non sembra alieno dall'identificare il contag io coleroso con minutissimi insetti impercettibili ad occhio nudo e prevede, sia pur vagamente, la funzione patogena delle tossine: «Ma anche morti (gli insetti) il lor principio deleterio come avviene della cantaride potrebbe agire sui centri nervosi, e cagionare i sintomi mortali, e la morte stessa» (p. 44-5). In tema di contagio, ricordo gli «esperimenti fatti sugli animali» durante l'epidemia oftalmica d'Ancona e precisamente la trasmissione della malattia mediante inoculazione del pus umano nel sacco congiuntivale e nell'uretra del cane. Sull'argomento ef. il lavoro di BELLoNi citato nella nota 40

${ }^{70}$ Cioè sul Giornale per servire ai progressi della Patologia e della Materia Medica si trovano i due lavori che hanno tramandato alla posterità il nome del Rima: Sulla cura radicale del varicocele, 4, 398-416 (1836). Sulla causa prossima delle varici alle estremità inferiori, e sulla loro cura radicale. Memoria letta nell'Ateneo di Venezia li 12 dicembre 1836 dal socio ordinario Tommaso Dott. Rima, 5, 265-301 (1836). I due lavori furono raccolti in un fascicolo separato: Cura radicale delle varici dedotta dalla causa prossima scoperta e dimostrata in due memorie lette nell' Ateneo dal socio ordinario Tommaso D. ${ }^{r}$ Rima, chirurgo primario anziano del Civico Ospitale Provinciale di Venezia, già chirurgo principale d'armata, professore di clinica e chirurgo in capo degli ospitali militari del cessato Regno d'Italia, premiato con la medaglia d'oro di S.M.I.R. Ferdinando Primo dal R. Istituto per l'invenzione dei bagni galleggianti fissi, o vagenti, ec. Edizione seconda con appendice dell'Autore, Venezia (G. Antonelli) 1838, di p. 60. Nell'appendice di questo scritto (p. 58-60) sono riferiti tre nuovi casi felicemente operati dal Riмa, nel terzo dei quali il circolo refluo era di un'evidenza lampante: «Giacendo anche orizzontalmente in letto, senza premettere vuotamento o compressione alla parte superiore della coscia, era visibile la refluenza del sangue. Poco rilevata la vena, sotto il più leggier conato di tosse espressamente promossa, inturgidiva e si tingeva prontamente d'un color ceruleo (essendo bianchissima la cute) e si vedeva il sangue discendere lungo la coscia. Era paragonabile in certo modo alla folgore che serpegçiando in un baleno si vede strisciare in cielo per un tratto lunghissimo. Non altrimenti e quasi con la stessa velocità prorompeva il 
sangue del tronco alle diramazioni. Sangue a rivi spruzzava da entrambe le estremità allorquando col ferro fu recisa la continuità del vaso.» Il primo dei tre nuovi casi dell'appendice del Rima era già stato pubblicato da M. Asson: Ulcera varicosa ad una gamba; escisione della vena safena posteriore al poplite; guarigione, Commentarii di Medicina (opera periodica di G. F. Spongia, Padova) 4, 484-6 (1837). Sullo stesso volume (p. 235-40 e 367-71) della stessa rivista M. Asson aveva precedentemente riassunto i due lavori fondamentali del RrMa sul varicocele e sulle varici, che furono inoltre riprodotti integralmente a p. 698-707 e 666-81 del t. 48 (1851) del Dizionario classico di medicina... di M.G. Levi . . Cf. inoltre le nostre note 1, 2 e 3. Le comunicazioni svolte dal Rima nel 1836 furono riassunte a p. 75-6 da G. NAMIAs, Dei lavori fatti dalla sezione per le scienze nell'anno accademico 1836-37. Relazione del Dottor Giacinto Namias segretario della classe, Esercitazioni scientifiche e letterarie dell'Ateneo di Venezia 3, 73-84 (1839).

${ }^{71}$ In quella seduta dell'Ateneo Veneto, il Rima non riferì soltanto sulle varici, ma anche sopra un suo brillante successo operatorio in altro campo: Storia di un voluminoso tumore estirpato dal collo di Marianna Talenti nel civico ospitale Provinciale di Venezia dal chirurgo in capo del medesimo ospitale Tommaso G. Rima ..., Giornale sulle Scienze e Lettere delle Provincie Venete (Treviso) 10, 213-23 (1826), 1 tav. (estratto di p. 16 e 1 tav. con titolo lievemente variato), dove leggiamo che «La presente narrazione $f u$ il soggetto di una lettura all'Ateneo di Venezia nella tornata accademica del 29 dicembre 1825 ...» (p. 223) e che «I cenni pratici sulla cura radicale delle varici alle gambe e sul pervertito ordine di circolazione letti in appendice della medesima (storia), saranno pubblicati separatamente» (p. 213, nota 1 ).

Un riassunto della Storia di un voluminoso tumore estirpato ... fu poi pubblicato, assieme alla succinta descrizione di due altre brillanti e felici interventi (asportazione di un enorme tumore pendulo del collo, estrazione d'un grosso palo penetrato nella regione occipitale), nelle Tavole con cenni istorici d'alcuni casi chirurgici straordinarj felicemente curati nel Civico Ospitale Provinciale di Venezia dal chirurgo primario anziano Tommaso Dott. Rima, cui fan seguito due memorie sulla causa prossima e sulla cura radicale delle varici, ec. Venezia (G. Antonelli) 1838, di p. 13 e tav. 3. Tali Tavole con cenni istorici... costituiscono talvolta un fascicolo a sè stante oppure possono essere unite in fascicolo unico (ma con numerazioni distinte) assieme alla Cura radicale delle varici . . Edizione seconda ... (1838, ef. nota 70) o a qualche lavoro minore del Rima.

${ }^{72}$ Lo scritto sull'ernia incarcerata, apparso sulla Gazzetta Privilegiata di Venezia del 24. aprile 1837, è diretto in forma epistolare a Luigi Valeriano Brera (1772-1840), che sull'argomento aveva riferito nel numero dell'11 aprile 1837, intrattenendosi particolarmente sopra un artificio (sottrazione dei gas intestinali) felicemente attuato da JAMES O' Beirne (1796-1862). Il Brera rispose poi al Rima nel numero del 3 maggio 1837.

${ }^{73}$ Il «giornale del Namias» (Giacinto Namias, 1810-1874) è il Giornale per servire ai progressi della Patologia e della Materia Medica fondato a Venezia nel 1834 dal Namias e da Maurizio Bufalini (1787-1875) e trasformatosi a partire dal vol. VIII (1838) nel Giornale per servire ai progressi della Patologia e della Terapeutica. In esso il Rima pubblicò le seguenti recensioni: Fasciature con cemento di amido pei fratturati proposte dal dottor Seutin 7, 568-9 (1837); Del varicocele o cirsocele, 8, 110-2 (1838); Memoria del dottor Jeanselme sui tumori bianchi estratta dalle lezioni cliniche del professor Velpeau, 8, 250-64 (1838); Apparecchi permanenti nelle fratture, 8, 476-7 (1838); Correzioni al 
metodo di Chopart per la disarticolazione parziale del piede, 9, 137-9 (1838); Nuovi processi operativi del dott. Petrequin in alcune malattie d'occhi, 9, 518-29 (1838).

${ }^{74}$ L. Belloni, I bagni galleggianti d'un chirurgo dell'Ottocento, Rivista Ciba 2 (fasc. 10), 343-4 (1948).

${ }^{75}$ Bagni galleggianti in Venezia privilegiati da S.M. l'Imperatore e Re Francesco I., premiati con la medaglia d'oro dal R. Istituto Italiano, estratti dal Supplemento del Nuovo Dizionario Tecnologico o di Arti e Mestieri, ec., che si pubblica in Venezia dall'ed. Giuseppe Antonelli. Senza luogo nè data, di p. I-VIII e 1 tav. ripiegata. Il testo corrisponde a quello del capitolo «Bagni sulle barche» (p. 119-125) della voce «Bagni» (da p. 114 a p. 128, dove tra i nomi degli autori figura anche quello del RrMA) del II vol. del Supplimento al nuovo dizionario universale tecnologico o di arti e mestieri compilato sulle migliori opere di scienze ed arti pubblicatesi negli ultimi tempi, e particolarmente su quelle di Berzelio, Dumas, Chevreul, Gay-Lussac, Hachette, Clement, Borgnis, Tredgold, Buchanam, Rees; dal Dizionario di Storia naturale, e da quello dell' Industria ec., ed esteso a ciò che più particolarmente può risguardare l'Italia, corrispondente al tomo 16 (1835) del Nuovo dizionario universale tecnologico o di arti e mestieri e della economia industriale e commerciante compilato dai signori Lenormand, Payen, Molard Jeune, Laugier, Francœur, Robiquet, Dufresnoy, ec. Prima traduzione italiana fatta da una società di dotti ed artisti, con l'aggiunta ...., Venezia (Giuseppe Antonelli editore) 1830-1859, di vol. 61.

${ }^{76}$ Collezione degli atti delle solenni distribuzioni de' premj d'industria fatte in Milano ed in Venezia dall'anno 1833 al 1839 (Milano) 6, 33-4 e 143-4 (1839).

77 Aggiunto nell'interlinea «(egli diceva)».

${ }^{78}$ François-Marie-Auguste Caffarelli (1766-1849).

79 «E il coltello anatomico spiò i guasti pertanto, e rivelò la causa vera del lungo e complicato malore, verificando in gran parte la diagnosi ch'ei ne avea fatta, perchè maggiore stima si meritasse e maggior compassione al pensiero delle tante e gravi sue sofferenze» declamò L. NARdo (Tributo alla memoria ...., p. 11) e aggiunse in nota «Moriva Tommaso Antonio Rima il 26 febbraio 1843, alle 23/4 pom., dopo quasi sedici mesi di decubito, nell'età d'anni 67 compiuti, in conseguenza di gravi lesioni degli organi uropojetici, di lenta artero-litiasi e paraplegia, cui erasi associata l'amaurosi. Colla necroscopia si riscontrò estesa ossificazione nelle arterie maggiori toraciche, nelle encefaliche e nelle ottalmiche, cui specialmente si attribuì la causa dell'amaurosi. Gli organi uropojetici si rinvennero essi pure molto malconci.»

${ }^{80}$ Elisa, madre di Orazio Pinelli, il quale ebbe da M. Asson il manoscritto della Necrologia e lo trasmise a D. Giordano (cf. nota 9).

${ }^{81}$ Enrichetta.

${ }^{82}$ Della resecazione della mascella inferiore affetta da osteo-sarcoma, operata dal dott. Rima chirurgo primario anziano del Civico Ospitale di Venezia. Relazione del dottor Antonio Marconi, medico-chirurgo secondario nel medesimo, letta all'Ateneo di Venezia li 22 aprile 1839, Giornale per servire ai progressi della Patologia e della Terapeutica 10, 198-212 (1839). Sullo stesso Giornale 12, 147-8 (1840), apparve anche una Lettera del Dott. Tommaso Rima al Dott. Giacinto Namias (Venezia, 18 dicembre 1839), in cui è descritto il mantenimento della guarigione alla distanza di dieci mesi. Il caso fu anche descritto nella Lettera del dott. Giacinto Namias al Compilatore della Gazztta (Venezia, 31 marzo 1839), Gazzetta Privilegiata di Venezia del 5 aprile 1839, n. 77, p. 306-7. Sotto il titolo 
Della resecazione della mandibola inferiore operata dal Prof. D.r Tommaso Rima in Venezia. Sunto storico della Relazione del dott. A. Marconi ..., apparve una breve descrizione del caso sia in fascicolo a parte (di p. 4 e tav. 1 , senza luogo nè data; spesso unito, ma con numerazione distinta, alla descrizione del caso successivo), che nella Gazzetta Ticinese del 7 agosto 1840, n. 32 , p. 277.

${ }^{83}$ Il caso fu descritto nella Lettera del dott. Carlo Mayer al dott. G. Namias (Mestre 4 novembre 1839), Gazzetta Privilegiata di Venezia dell'8 novembre 1839, n. 256, p. 1024. Sotto il titolo Operazione cesarea con felicissimo successo eseguita dal Profess. Dott. Tommaso Rima già pubblicata nella Gazzetta Privilegiata di Venezia ... la relazione del Mayer apparve anche in fascicolo a parte (di p. 3, senza luogo nè data; spesso unito, ma con numerazione distinta, alla descrizione del caso precedente) e nella Gazzetta Ticinese del 10 agosto 1840, suppl. n. 32, p. 283.

Altri due brillanti interventi compiuti dal Rima negli ultimi anni di vita sono segnalati da M. Asson (Elogio ..., p. 325).

${ }^{84} \mathrm{Fu}$ coronato da menzione onorevole nella distribuzione de' premii d'industria dell'anno 1842 fattasi in Venezia: «Dott. Tommaso Rima di Venezia. Siringa a dardo. Questa siringa prodotta dal dott. Rima fu giudicata un miglioramento di quella dello Scarpa per la operazione ipogastrica della pietra. Essa consiste in un tubo cilindrico curvato all'apice, da cui, mediante una molla articolata, spingendo esce il dardo feritore della vescica. Parecchi furono i vantaggi riscontrati in questa siringa sopra quella dello SCARPA, per cui fu al suo autore aggiudicata la menzione onorevole» (Collezione degli atti delle solenni distribuzioni de' premj d'industria fatte in Milano ed in Venezia dall'anno 1840 al 1852, Venezia, 7, 140-1, 1852). Lo strumento è descritto anche da M. Asson (Elogio ..., p. 323): «Ed ancora di perfezionare il taglio ipogastrico per la cistotomia egli si propose con una sciringa nella quale, insieme al dardo feritore della vescica, esce un sostegno che la ripiegatura peritoneale dal rimaner lesa protegge. Offre pure tale stromento il vantaggio di poter spingere le injezioni nella vescica, ed essendo formato di vari pezzi può venire decomposto in modo che ne riesca agevole, quando necessiti, l'estrazione per la via della ferita ipogastrica.» L'Asson aveva precedentemente menzionato che «Un suo strumento per la cura delle fistole cisto-vaginali può essere vantaggiosamente applicato in quelle che sono prossime all'orifizio vaginale, è ingegnosissimo, più semplice che quelli inventati dai celebri LaLlemand e Dupurtren, e meno molesto.» I due strumenti sono menzionati anche da L. NARDo (Tributo alla memoria ..., p. 15): «Nel 1840 inventava un Catetere articolato a dardo per il taglio ipogastrico per l'estrazione dei calcoli vescicali. Altro istromento immaginava per la cura della fistola uretrovaginale e per l'incontinenza dell'urina nelle donne. D'entrambi questi istromenti non potè offrire però utili pratici esperimenti, stante la sopravenutagli malattia.» 